\title{
Macro-scale models for fluid flow in tumour tissues: impact of microstructure properties
}

\author{
Cristina Vaghi ${ }^{1}$, Raphaelle Fanciullino ${ }^{2}$, Sebastien Benzekry ${ }^{1}$, Clair Poignard ${ }^{1}$ \\ ${ }^{1}$ Team MONC, Inria, Institut de Mathématiques de Bordeaux, CNRS, Bordeaux INP, Univ. Bordeaux, France \\ ${ }^{2}$ SMARTc, CRCM Inserm UMR1068, CNRS UMR7258, Aix Marseille University, France
}

\begin{abstract}
Understanding the dynamics underlying fluid transport in tumour tissues is of fundamental importance to assess processes of drug delivery. Here, we analyse the impact of the tumour microscopic properties on the macroscopic dynamics of vascular and interstitial fluid flow by using formal asymptotic techniques.

Here, we obtained different macroscopic continuum models that couple vascular and interstitial flows. The homogenization technique allows us to derive two macroscale tissue models of fluid flow that take into account the microscopic structure of the vessels and the interstitial tissue. Different regimes were derived according to the magnitude of the vessel wall permeability and the interstitial hydraulic conductivity. Importantly, we provide an analysis of the properties of the models and show the link between them. Numerical simulations were eventually performed to test the models and to investigate the impact of the microstructure on the fluid transport.

Future applications of our models include their calibration with real imaging data to investigate the impact of the tumour microenvironment on drug delivery.
\end{abstract}

Keywords: two-scale homogenisation, fluid flow in tumours, interstitial fluid pressure, tumour microscopic structure.

\section{Introduction}

Interstitial and capillary fluids are strongly connected in malignant tissues and are mainly involved in the transport of molecules in tumours. When drugs are intravenously injected, they have to overcome several barriers, including vascular transport, transvascular transfer, interstitial transport and finally cellular uptake [1]. The biological and physicochemical properties of the tumour microenvironment play a significant role in the drug delivery process [2]. The geometrical microstructure of the tumour also has an important impact on the fluid flow [3].

Neoplastic tissues are highly heterogeneous. They are generally characterized by [4] accumulated solid stress [5], abnormal blood vessels network [6], elevated interstitial fluid pressure (IFP) [7], that almost equals the microvessel pressure (MVP) and dense interstitial structure [8]. These traits, that distinguish tumour tissues from normal ones, cause barriers to drug delivery [1]. The heterogeneous spatial distribution of tumour vessels and poor lymphatic drainage impair a uniform delivery of therapeutic agents in tumours. Blood vessels are unevenly distributed, leaving avascular spaces. Moreover, their walls are leaky and hyperpermeable in some places while not in other [9]. Blood flow velocity is also compromised by the elevated viscous and geometrical resistance offered by the tumour vasculature 
[3]. Finally, the lack of an efficient lymphatic network inside the tumour coupled with leaky tumour vessels leads to a high IFP [10] almost equal to the microvascular pressure [7]. Due to elevated IFP, the tumour interstitium is characterized by no pressure gradient $[11,12]$.

Several mathematical models have been developed during the last decades to investigate the features of fluid transport in the tumour microenvironment. The porous medium theory has been employed to model interstitial fluid flow (IFF) relying on Darcy's law and using average field variables defined over the whole tissue $[13,14]$. Fluid transport through the blood vessels has been exploited in both discrete and continuous manners, including spatial and temporal variations. In either discrete and continuous models, the IFF and microvascular fluid (MVF) are usually coupled by Starling's law [15], that describes the fluid filtration through the highly permeable vessels walls. Microscopic models of the flow patterns around an individual capillary and a network of blood vessels have been introduced relying on the Krogh cylinder model $[16,17,18]$. Poiseuille's law can be considered to describe the blood flow in a cylindrical domain [19, 20, 21]. Furthermore, Navier-Stokes equations have been adopted to model the spatio-temporal variations in blood flow $[18,14]$. More detailed biophysical models have been developed to take into account the more realistic heterogeneity of the tumour vasculature [22]. Welter et al [23] introduced an exhaustive biophysical model the incorporates tumor growth, vascular network (including arteries and veins), angiogenesis, vascular remodeling, porous medium description for the extracellular matrix (ECM) and interstitial fluid, interstitial fluid pressure and velocity and chemical entities (such as oxygen, nutrients, drugs). On the other hand, continuous models based on mixture theory have been exploited to describe interstitial and vascular fluid flow, assuming that the two phases are present at each point of the tumour [24]. Multiscale models have further been employed to investigate the coupling between tumor growth, angiogenesis, vascular remodelling and fluid transport [25] and the impact of collagen microstructure on interstitial fluid flow [26]. Imaging data have been integrated to both continuum and discrete models to quantify the effect of the heterogeneity on the fluid transport [27, 28].

The increasing amount of imaging data makes it possible to recover vascular networks in details. However, solving discrete models on the entire vessel tree might be computationally expensive. The formal two-scale homogenization technique allows to take into account microscopic features on the macroscopic dynamic of fluid flow. Two-scale asymptotic expansion has been previously applied to fluid and drug transport in tumours. A system of Darcy's equations has been derived in [29] to couple interstitial and vascular fluid flows in malignant tissues assuming a periodic medium. A higher complexity has been taken into account in [30], with the introduction of rheological effects in the blood flow and of local heterogeneity. A generalization of homogenized modelling for vascularized poroelastic materials has also been presented [31, 32]. More recently, higher complexity has been added to the homogenized models [33] considering three length scales for the vessel network (i.e., arteriole, venule and capillary scales).

The main novelty of this work is the study of the impact of the tumour microscopic properties on the global fluid dynamic. First, we describe a system of partial differential equations coupling interstitial, transvascular and capillary flows at the microscopic scale. While the interstitium and the capillary walls are assumed to be porous media where the fluid is governed by Darcy's law, the blood in the capillaries is considered a Newtonian fluid described by the Stokes equation. As the thickness of the capillary walls tends to zero, an asymptotic analysis similar to $[34,35]$ enables us to derive a Starling's 
law-type equation across the vessel wall for the transvascular transport of fluid flow. Then, we perform a two-scale analysis under periodic assumption [36] to derive formally effective macroscale tissue models of fluid flow for 3 asymptotic regimes depending on the magnitude of the permeability of the vessel wall and of the interstitial hydraulic conductivity. These models combine the effects of the interstitial compartment and the capillaries at the microscale, providing thus a link between the microstructure and the macroscopic fluid flow. Moreover, we compare the different asymptotic regimes with some models given in literature (namely, [2], [29], [30]) and show the links between the different models. In particular, we show that for the model initially derived by Shipley and Chapman [29] the difference between the capillary and the interstitium pressures decays exponentially fast from the boundary, making thus a link with the Baxter and Jain model [2]. Furthermore, we present the mathematical and numerical analysis on the homogenised tensors in order to assess their properties according to the geometric microstructure. Eventually, numerical simulations on the macroscopic models are performed and the results are compared to the literature.

This approach can be applied to study the impact of the tumour microscopic characteristics on drug delivery. Imaging data can provide the tissue microstructure that can be integrated in the homogenised model. This modelling technique prevents the resolution of the original micro-scale model that might be unfeasible as it requires the discretisation of the entire vessel network and porous medium. Moreover, the heterogeneities of malignant tissues can be taken into account by considering the spatial variability of the micro-vessel features at the macroscopic scale.

\section{Main results}

First, we motivated the interface conditions between the interstitial compartment and the capillaries of the micro-scale model using an asymptotic expansion technique. We obtained a Starling's law type equation to describe the flux through the vessel walls. Moreover, we considered the Joseph-BeaversSaffman slip condition at the boundary between the capillary lumen and the vessel wall. This condition states that the slip velocity along the vessel wall is proportional to the shear stress [37].

Then, using the two-scale asymptotic homogenisation, we assumed that a generic variable $v^{\varepsilon}(\mathbf{x})$, as function of the macroscopic spatial variable $\mathbf{x}$ and of the microscopic parameter $\varepsilon$, could be approximated as

$$
v^{\varepsilon}(\mathbf{x})=v(\mathbf{x})+\varepsilon \phi\left(\frac{\mathbf{x}}{\varepsilon}\right) \nabla_{\mathbf{x}} v(\mathbf{x}),
$$

where $v(\mathbf{x})$ is the macroscopic variable and $\phi\left(\frac{\mathbf{x}}{\varepsilon}\right)$ is the corrector that takes into account oscillations at the microscopic scale. Using this approach, we derived three different macroscale models to describe the fluid transport in tumour tissues according to the magnitude of the permeability of the vessel walls and of the interstitial hydraulic conductivity. In particular, the following regimens were derived for the interstitial fluid pressure $p_{t}$ and the capillary pressure $p_{c}$ :

- Model 1: highly permeable walls and large interstitial hydraulic conductivity

$$
\nabla \cdot\left(\left(\mathbf{K}+\frac{C_{t}}{C_{c}} \mathbf{E}\right) \nabla p_{c}\right)=0 \quad p_{t}=p_{c} .
$$


- Model 2: weakly permeable walls and large interstitial hydraulic conductivity

$$
\nabla \cdot\left(\mathbf{K} \nabla p_{t}\right)=C_{t}\left(p_{t}-p_{c}\right), \quad \nabla \cdot\left(\mathbf{E} \nabla p_{c}\right)=C_{c}\left(p_{c}-p_{t}\right)
$$

- Model 3: weakly permeable walls and small interstitial hydraulic conductivity

$$
\nabla \cdot\left(\mathbf{K} \nabla p_{t}\right)=C_{t}\left(p_{t}-p_{c}\right), \quad \nabla \cdot\left(\mathbf{E} \nabla p_{c}\right)=0
$$

In the three models, $\mathbf{K}$ and $\mathbf{E}$ are the permeability tensors of the interstitium and the capillaries, respectively, and $C_{t}, C_{c}$ are constant parameters that will be defined later on. The definition of these tensors involves the so-called correctors, as usual in homogenisation. It characterises the impact of the microstructure on the effective properties of the tissue at the macroscale. Model (2) has been derived assuming a small magnitude of the capillary permeability and confirms previous results [29, 30].

The interstitial fluid flow $\mathbf{u}_{t}$ and the blood velocity $\mathbf{u}_{c}$ are given in the first two cases by the average quantities

$$
\mathbf{u}_{t}=-\mathbf{K} \nabla p_{t}, \quad \mathbf{u}_{c}=-\mathbf{E} \nabla p_{c},
$$

while for the third model they are given by

$$
\mathbf{u}_{t}=-\varepsilon \mathbf{K} \nabla p_{t}, \quad \mathbf{u}_{c}=-\mathbf{E} \nabla p_{c} .
$$

Boundary conditions must be added in order to guarantee well-posedness. Dirichlet-Dirichlet boundary conditions can be imposed to $p_{t}$ and $p_{c}$ if the pressures in the sourranding tissue are known. Mixed Dirichlet and Neumann boundary conditions can be chosen to ensure the continuity of $\mathbf{u}_{t}$ or $\mathbf{u}_{c}$.

\section{Microscale model of fluid transport in tumours}

At the microscale, the domain $\Omega \in \mathbb{R}^{N}$ (with $N=2,3$ ) is the medium that consists of the interstitium $\Omega_{t}$ and the capillary region $\Omega_{c}$ (Fig. 1). The interface between the capillary and the vessel wall and the one between the interstitium and the vessel wall are denoted respectively by $\Gamma=\partial \Omega_{c} \cap \partial \Omega_{m}$. In the two regions, the fluid flow is assumed to be incompressible.

The interstitium - composed by the cells and the extracellular matrix and collagen - is modeled as an isotropic porous medium, where the velocity $\mathbf{u}_{t}$ and pressure $p_{t}$ follow the Darcy's law:

$$
\nabla \cdot \mathbf{u}_{t}=0, \quad \mathbf{u}_{t}=-k_{t} \nabla p_{t} \quad \text { in } \Omega_{t}
$$

where $k_{t}$ is the hydraulic conductivity in the interstitium.

In the capillaries, we assume that the fluid is Newtonian with a constant viscosity $\mu$. Neglecting the inertial effects and under the assumption of a laminar flow, we end up with the Stokes equation for the description of the vessel velocity $\mathbf{u}_{c}$ and pressure $p_{c}$

$$
\nabla \cdot \mathbf{u}_{c}=0, \quad \mu \nabla^{2} \mathbf{u}_{c}=\nabla p_{c} \quad \text { in } \Omega_{c} .
$$



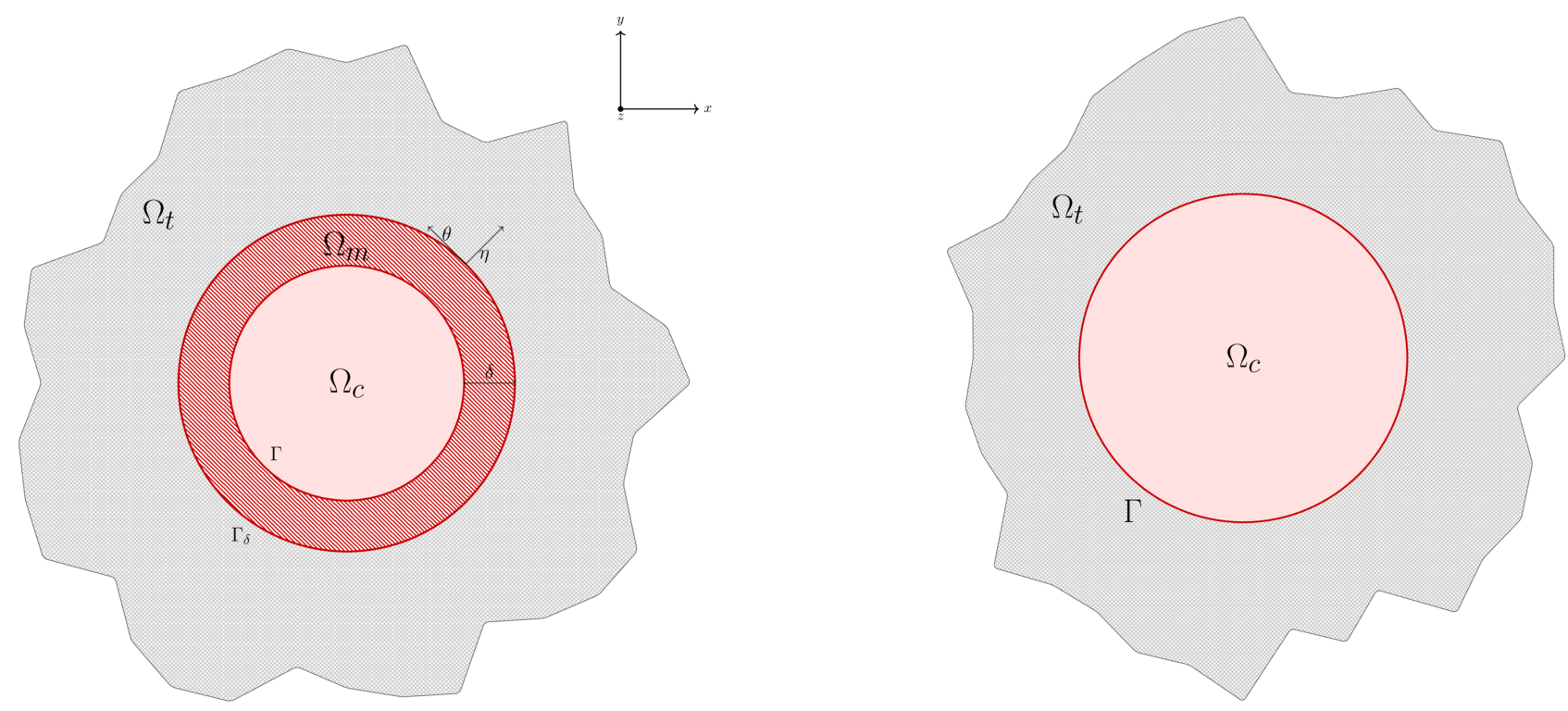

Figure 1. Left: schematic of the domain considered to compute the interface conditions between the capillaries and the interstitium. $\Omega_{m}$ denotes the vessel wall region, $\Gamma_{\delta}$ is the interface between the vessel wall and the interstitium. The transmission conditions are derived as $\delta \rightarrow 0$ using asymptotic expansion. Right: schematic representation of the domain of the microscopic model: section of the capillary in the sourranding tissue.

\section{Interface conditions}

Along the vessel wall $\Gamma$, interface conditions couple the different equations in the two domains. We performed an asymptotic expansion in order to derive suitable interface conditions between the capillaries and the interstitial compartment as shown in Supplementary Information S1. We considered the vessel membrane as a thin and weakly porous medium and obtained the following interface conditions from the thin layer model, getting similar results as [29, 30]:

1. Continuity of the normal velocity:

$$
\mathbf{u}_{c} \cdot \mathbf{n}=\mathbf{u}_{t} \cdot \mathbf{n} \quad \text { on } \Gamma
$$

This condition guarantees the continuity of mass through the interface and it is a natural choice since the fluid is assumed to be incompressible in the two regions.

2. Starling 's law-type equation, that is the most widely used equation in literature to model flux transport across the vessel wall $[12,19]$ :

$$
\mathbf{u}_{c} \cdot \mathbf{n}=L_{p}\left(p_{c}-p_{t}-\mu\left[(\mathbf{n} \cdot \nabla) \mathbf{u}_{c}\right] \cdot \mathbf{n}\right) \quad \text { on } \Gamma
$$

where $L_{p}$ is the vascular permeability. The difference between the osmotic pressures was considered negligible compared to the interstitial fluid pressure difference in tumours [13, 38]. Moreover, the viscous term in equation (5) is usually neglected but it is necessary to guarantee the wellposedness of the problem and does not change the physical meaning since it is based on the 
balance of the normal forces [30].

3. Beavers-Joseph-Saffmann condition on the tangential component of the capillary velocity at the boundary with a porous medium:

$$
\mathbf{u}_{c} \cdot \boldsymbol{\tau}=-\frac{\sqrt{k_{m} \mu}}{\alpha_{\mathrm{BJ}}}\left[(\mathbf{n} \cdot \nabla) \mathbf{u}_{c}\right] \cdot \boldsymbol{\tau} \quad \text { on } \Gamma
$$

where $\alpha_{\mathrm{BJ}}$ is a constant depending on the properties of the interface and $k_{m}$ is the hydraulic conductivity of the vessel walls. This condition comes from the experimental evidence shown by Beavers and Joseph [37] who observed that the slip velocity along $\Gamma$ was proportional to the shear stress along $\Gamma$. Equation of the form (6) was derived by Saffmann using a statistical approach and the Brinkman approximation for non-homogeneous porous medium [39] .

\section{Non-dimensionalization}

We perform a dimensional analysis in order to understand the relative amplitude of the different parameters involved. We rescale our fields as follows:

$$
\mathbf{x}=L \mathbf{x}^{\prime}, \quad \mathbf{u}=U \mathbf{u}^{\prime}, \quad p=\frac{\mu L U}{d^{2}} p^{\prime}+p_{0},
$$

where $L$ is the characteristic domain length, $d$ is the mean intercapillary distance and $U$ is a characteristic velocity. The non-dimensional fluid transport problem reads (neglecting the primes for the sake of simplicity)

$$
\begin{aligned}
& \nu \nabla^{2} \mathbf{u}_{c}=\nabla p_{c}, \quad \nabla \cdot \mathbf{u}_{c}=0, \quad \text { in } \Omega_{c}, \\
& \mathbf{u}_{t}=-\kappa \nabla p_{c}, \quad \nabla \cdot \mathbf{u}_{t}=0, \quad \text { in } \Omega_{t}, \\
& \mathbf{u}_{c} \cdot \mathbf{n}=\mathbf{u}_{t} \cdot \mathbf{n} \quad \text { on } \Gamma \text {, } \\
& \mathbf{u}_{c} \cdot \mathbf{n}=R_{\mathbf{n}}\left(p_{c}-p_{t}-\nu\left[(\mathbf{n} \cdot \nabla) \mathbf{u}_{c}\right] \cdot \mathbf{n}\right) \quad \text { on } \Gamma \text {, } \\
& \mathbf{u}_{c} \cdot \boldsymbol{\tau}=-R_{\boldsymbol{\tau}}\left[(\mathbf{n} \cdot \nabla) \mathbf{u}_{c}\right] \cdot \boldsymbol{\tau}, \quad \text { on } \Gamma \text {. }
\end{aligned}
$$

where

$$
\nu=\frac{d^{2}}{L^{2}}, \quad \kappa=\frac{k_{t} \mu}{d^{2}}, \quad R_{\mathbf{n}}=\frac{L_{p} L \mu}{d^{2}}, \quad R_{\tau}=\frac{\sqrt{k_{m} \mu}}{\alpha_{\mathrm{BJ}} L},
$$

are dimensionless quantities.

\section{Continuum macroscale models using two scale asymptotic analysis}

This section is devoted to the derivation of a continuum macro-scale model using the two scale asymptotic expansion method $[40,41,36]$. We assume that $d$ is the mean inter-capillary distance and $L$ is the tissue characteristic length such that $\varepsilon=d / L \ll 1$. We denote by $Y$ the reference periodic cell that is contained in $[0,1]^{N}$ and is composed by the interstitium $Y_{t}$ and the capillaries $Y_{c}$, i.e. $Y=Y_{c} \cup Y_{t}$ and the interface $\Gamma_{Y}=\partial Y_{c} \cap \Gamma_{Y_{t}}$. We assume that the interface is entirely contained in $Y$, i.e. $\Gamma_{Y} \cap \partial Y=\emptyset$. The normal vector $\mathbf{n}$ to the interface $\Gamma_{Y}$ is directed outward the vascular domain 
A

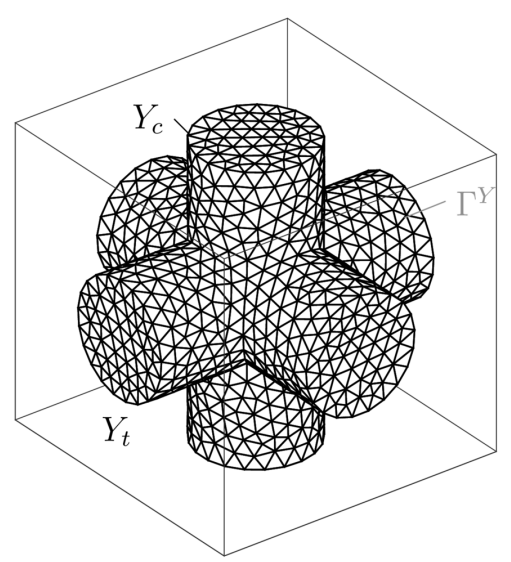

B

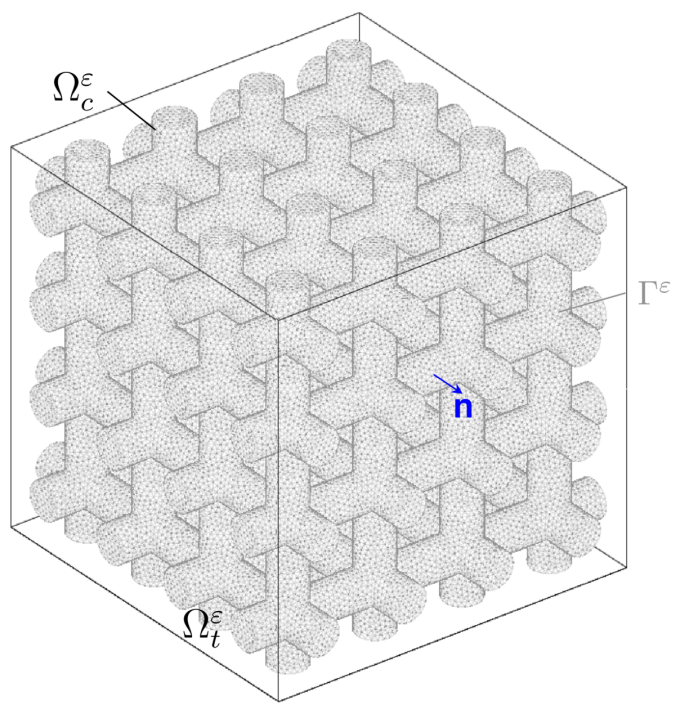

Figure 2. Unitary cell $Y=[0,1]^{3}$ (left) composed by the capillary region $Y_{c}$ and the interstitial compartment $Y_{t}$; the interface between the two regions is denoted by $\Gamma_{Y}$. Periodic domain $\Omega$ (right): the tumour capillaries $\Omega_{c}^{\varepsilon}$ are assumed to be in the tubes, while the outer region corresponds to the interstitial compartment $\Omega_{t}^{\varepsilon}$; the interface between the two regions is denoted by $\Gamma^{\varepsilon}$ and the normal $\mathbf{n}$ is directed outward the vascular domain.

$Y_{c}$. The total domain $\Omega$ is divided periodically in each direction in identical squares $Y_{n}^{\varepsilon}$ such that

$Y_{n}^{\varepsilon}=\varepsilon n+\varepsilon Y, \quad Y_{t, n}^{\varepsilon}=\varepsilon n+\varepsilon Y_{t}, \quad Y_{c, n}^{\varepsilon}=\varepsilon n+\varepsilon Y_{c}, \quad \Gamma_{n}^{\varepsilon}=\varepsilon n+\varepsilon \Gamma_{Y}, \quad \forall n \in\left\{i \in \mathbb{Z}^{N} \mid Y_{i}^{\varepsilon} \cap \Omega \neq \emptyset\right\}$.

Therefore, the domain $\Omega$ is composed of two subdomains $\Omega_{t}^{\varepsilon}=\cup_{n} Y_{t, n}^{\varepsilon}$ and $\Omega_{c}^{\varepsilon}=\cup_{n} Y_{c, n}^{\varepsilon}$ that depend on $\varepsilon$ and are connected when $N=3$. The interface between the two subdomains is $\Gamma^{\varepsilon}=\cup_{n} \Gamma_{n}^{\varepsilon}$. Figure 2 shows a schematic illustration of the periodic domain and of the unitary cell $Y$. According to the multiple scales theory, we introduce a spatial variation decoupling

$$
\mathbf{y}=\frac{\mathbf{x}}{\varepsilon},
$$

and assume that the macro and the micro spatial variables ( $\mathbf{x}$ and $\mathbf{y}$, respectively) are independent. Therefore, any field $g$ that we have introduced before ( $\operatorname{such}$ as $\mathbf{u}_{t}, \mathbf{u}_{c}, p_{t}, p_{c}$ ) depends on $\varepsilon$ and is assumed to be written following an asymptotic expansion as:

$$
g=g^{\varepsilon}(\mathbf{x}, \mathbf{y})=\sum_{l=0}^{\infty} \varepsilon^{l} g^{(l)}(\mathbf{x}, \mathbf{y})
$$

where $g^{(l)}(\mathbf{x}, \mathbf{y})$ are $Y$-periodic functions.

Assuming that $\nu$ is of the same scale of $\varepsilon^{2}$, that $R_{\mathbf{n}}$ is of the order of $\varepsilon^{\gamma}$ (with $\gamma=0,1,2$ ) and that $\kappa$ is of the order of $\varepsilon^{\eta}$ (with $\eta=0,1$ ), we rescale the three parameters as $\nu=\varepsilon^{2} \bar{\nu}, R_{\mathbf{n}}=\varepsilon^{\gamma} \bar{R}_{\mathbf{n}}$ and $\kappa=\varepsilon^{\eta} \bar{\kappa}$ (so that $\bar{\nu}=\mathcal{O}(1), \bar{R}_{\mathbf{n}}=\mathcal{O}(1)$ and $\left.\bar{\kappa}=\mathcal{O}(1)\right)$. The choice of the rescaling of the parameter 
$\nu$ is made in order to avoid trivial solution when $\varepsilon \rightarrow 0$. We analyse the different regimens on varying the exponent $\gamma$.

We summarize here the main results relative to the leading order and derive formally the homogenized models in the Supporting Information S2. Let us denote by $\left|Y_{c}\right|=\int_{Y_{c}} d \mathbf{y},\left|Y_{t}\right|=\int_{Y_{t}} d \mathbf{y}$ and $\left|\Gamma_{Y}\right|=\int_{\Gamma_{Y}} d s$ the measures of the subdomains $Y_{c}$ and $Y_{t}$ and of the interface $\Gamma_{Y}$, respectively. We introduce the following two cell problems on the $Y$-periodic cell variables $\mathrm{G}^{j}, \mathbf{W}_{j}$ and $\mathrm{P}_{j}(j=1, \ldots, N)$ :

$$
\begin{aligned}
& \nabla_{\mathbf{y}}^{2} \mathrm{G}^{j}=0 \quad \text { in } Y_{t} \\
& \nabla_{\mathbf{y}} \mathrm{G}^{j} \cdot \mathbf{n}=\mathbf{n} \cdot \mathbf{e}_{j} \quad \text { on } \Gamma_{Y} . \\
& \bar{\nu} \nabla_{\mathbf{y}}^{2} \mathbf{W}^{j}+\mathbf{e}_{j}=\nabla_{\mathbf{y}} \mathrm{P}^{j} \quad \text { in } Y_{c}, \\
& \nabla_{\mathbf{y}} \cdot \mathbf{W}^{j}=0 \quad \text { in } Y_{c}, \\
& \mathbf{W}^{j} \cdot \mathbf{n}=0 \quad \text { on } \Gamma_{Y}, \\
& {\left[\left(\mathbf{n} \cdot \nabla_{\mathbf{y}}\right) \mathbf{W}^{j}\right] \cdot \boldsymbol{\tau}=0 \quad \text { on } \Gamma_{Y} .}
\end{aligned}
$$

Let us define the following tensors:

$$
[\mathbf{K}]_{i j}=\delta_{i j}-\frac{1}{\left|Y_{t}\right|} \int_{Y_{t}} \nabla_{\mathbf{y}} \mathrm{G}^{j} \cdot \mathbf{e}_{i} d \mathbf{y}, \quad[\mathbf{E}]_{i j}=\frac{1}{\left|Y_{c}\right|} \int_{Y_{c}} \mathbf{W}_{j} \cdot \mathbf{e}_{i} d \mathbf{y} .
$$

The equations for the leading orders are summarized in Table 1. Boundary conditions need to be added, such as Dirichlet or Neumann conditions.

For the sake of simplicity, from now on we denote by $\left(p_{t}, p_{c}\right)$ the leading orders of the interstitial and capillary pressures $\left(p_{t}^{(0)}, p_{c}^{(0)}\right)$.

\subsection{Tensors properties}

In order to ensure the well-posedness of the models that we have derived, the permeability tensors $\mathbf{K}$ and $\mathbf{E}$ need to be positive definite. This section is devoted to the analysis of the tensor properties with respect to the periodic cell $Y$.

Lemma 3.1. The tensor $\boldsymbol{K}$ is symmetric and positive definite.

Proof. Thanks to the Lax-Milgram theorem, problem $(7)$ has a unique solution in $H^{1}\left(Y_{t}\right) / \mathbb{R}$. The variational formulation associated to (7) reads

$$
\int_{Y_{t}} \nabla_{\mathbf{y}} \mathrm{G}^{j} \cdot \nabla_{\mathbf{y}} \varphi d \mathbf{y}-\int_{\Gamma_{Y}} \mathbf{e}_{j} \cdot \mathbf{n}_{\text {out }} \varphi d s=0
$$

for any periodic $\varphi \in H^{1}\left(Y_{t}\right)$ such that $\langle\varphi\rangle_{Y_{t}}=0$. Considering $\varphi=\mathrm{G}^{i}$ on $Y_{t}$, the following equations 
(1) $\gamma=0, \eta=0$

$$
\begin{array}{rlrl}
p_{t}^{\varepsilon}(\mathbf{x}, \mathbf{y}) \rightarrow p_{t}^{(0)}(\mathbf{x}) & \nabla \cdot\left(\left(\bar{\kappa} \mathbf{K}+\frac{\left|Y_{c}\right|}{\left|Y_{t}\right|} \mathbf{E}\right) \nabla_{t}^{(0)}\right) & =0 \\
\mathbf{u}_{t}^{\varepsilon}(\mathbf{x}, \mathbf{y}) \rightarrow \mathbf{u}_{t}^{(0)}(\mathbf{x}, \mathbf{y}) & \left\langle\mathbf{u}_{t}^{(0)}\right\rangle_{Y_{t}} & =-\bar{\kappa} \mathbf{K} \nabla p_{t}^{(0)} \\
p_{c}^{\varepsilon}(\mathbf{x}, \mathbf{y}) \rightarrow p_{c}^{(0)}(\mathbf{x}) & p_{c}^{(0)}=p_{t}^{(0)} \\
\mathbf{u}_{c}^{\varepsilon}(\mathbf{x}, \mathbf{y}) \rightarrow \mathbf{u}_{c}^{(0)}(\mathbf{x}, \mathbf{y}) & \left\langle\mathbf{u}_{c}^{(0)}\right\rangle_{Y_{c}} & =-\mathbf{E} \nabla p_{c}^{(0)}
\end{array}
$$

$p_{t}^{\varepsilon}(\mathbf{x}, \mathbf{y}) \rightarrow p_{t}^{(0)}(\mathbf{x})$

$\mathbf{u}_{t}^{\varepsilon}(\mathbf{x}, \mathbf{y}) \rightarrow \mathbf{u}_{t}^{(0)}(\mathbf{x}, \mathbf{y})$

(2) $\gamma=1, \eta=0$

(3) $\gamma=2, \eta=1$

$$
\begin{aligned}
& p_{c}^{\varepsilon}(\mathbf{x}, \mathbf{y}) \rightarrow p_{c}^{(0)}(\mathbf{x}) \\
& \mathbf{u}_{c}^{\varepsilon}(\mathbf{x}, \mathbf{y}) \rightarrow \mathbf{u}_{c}^{(0)}(\mathbf{x}, \mathbf{y})
\end{aligned}
$$$$
\nabla \cdot\left(\bar{\kappa} \mathbf{K} \nabla p_{t}^{(0)}\right)=\frac{\bar{R}_{\mathbf{n}}\left|\Gamma_{Y}\right|}{\left|Y_{t}\right|}\left(p_{t}^{(0)}-p_{c}^{(0)}\right)
$$$$
\left\langle\mathbf{u}_{t}^{(0)}\right\rangle_{Y_{t}}=-\bar{\kappa} \mathbf{K} \nabla p_{t}^{(0)}
$$$$
\nabla \cdot\left(\mathbf{E} \nabla p_{c}^{(0)}\right)=\frac{\bar{R}_{\mathbf{n}}\left|\Gamma_{Y}\right|}{\left|Y_{c}\right|}\left(p_{c}^{(0)}-p_{t}^{(0)}\right)
$$$$
\left\langle\mathbf{u}_{c}^{(0)}\right\rangle_{Y_{c}}=-\mathbf{E} \nabla p_{c}^{(0)}
$$

$p_{t}^{\varepsilon}(\mathbf{x}, \mathbf{y}) \rightarrow p_{t}^{(0)}(\mathbf{x})$

$$
\begin{aligned}
\nabla \cdot\left(\bar{\kappa} \mathbf{K} \nabla p_{t}^{(0)}\right) & =\frac{\bar{R}_{\mathbf{n}}\left|\Gamma_{Y}\right|}{\left|Y_{t}\right|}\left(p_{t}^{(0)}-p_{c}^{(0)}\right) \\
\left\langle\mathbf{u}_{t}^{(0)}\right\rangle_{Y_{t}} & =-\varepsilon \bar{\kappa} \mathbf{K} \nabla p_{t}^{(0)}
\end{aligned}
$$

$$
\begin{aligned}
& p_{c}^{\varepsilon}(\mathbf{x}, \mathbf{y}) \rightarrow p_{c}^{(0)}(\mathbf{x}) \\
& \mathbf{u}_{c}^{\varepsilon}(\mathbf{x}, \mathbf{y}) \rightarrow \mathbf{u}_{c}^{(0)}(\mathbf{x}, \mathbf{y})
\end{aligned}
$$

$$
\left\langle\mathbf{u}_{c}^{(0)}\right\rangle_{Y_{c}}=-\mathbf{E} \nabla p_{c}^{(0)}
$$

Table 1. Main results of the two-scale asymptotic analysis on varying the order of the parameters $R_{\mathbf{n}}$ and $\kappa$. 
hold thanks to the divergence theorem

$$
\begin{aligned}
0 & =\int_{Y_{t}} \nabla_{\mathbf{y}} \mathrm{G}^{j} \cdot \nabla_{\mathbf{y}} \mathrm{G}^{i} d \mathbf{y}-\int_{\Gamma_{Y}} \mathbf{e}_{j} \cdot \mathbf{n}_{\text {out }} \mathrm{G}^{i} d s \\
& =\int_{Y_{t}} \nabla_{\mathbf{y}} \mathrm{G}^{j} \cdot \nabla_{\mathbf{y}} \mathrm{G}^{i} d \mathbf{y}-\int_{Y_{t}} \nabla_{\mathbf{y}} \cdot\left(\mathrm{G}^{i} \mathbf{e}_{j}\right) d \mathbf{y} \\
& =\int_{Y_{t}} \nabla_{\mathbf{y}} \mathrm{G}^{j} \cdot \nabla_{\mathbf{y}} \mathrm{G}^{i} d \mathbf{y}-\int_{Y_{t}} \nabla_{\mathbf{y}} \mathrm{G}^{i} \cdot \mathbf{e}_{j} d \mathbf{y} .
\end{aligned}
$$

Therefore, the tensor $\mathbf{K}$ can be rewritten as

$$
\begin{aligned}
{[\mathbf{K}]_{i j} } & =\delta_{i j}-\frac{1}{\left|Y_{t}\right|} \int_{Y_{t}} \nabla_{\mathbf{y}} \mathrm{G}^{j} \cdot \mathbf{e}_{i} d \mathbf{y} \\
& =\delta_{i j}-\frac{1}{\left|Y_{t}\right|} \int_{Y_{t}} \nabla_{\mathbf{y}} \mathrm{G}^{j} \cdot \nabla_{\mathbf{y}} \mathrm{G}^{i} d \mathbf{y}, \\
& =\frac{1}{Y_{t}} \int_{Y_{t}} \nabla_{\mathbf{y}}\left(\mathrm{G}^{i}-y_{i}\right) \nabla_{\mathbf{y}}\left(\mathrm{G}^{j}-y_{j}\right) d \mathbf{y} .
\end{aligned}
$$

It follows that the tensor $\mathbf{K}$ is symmetric. To prove that the tensor is positive definite, we consider any $\boldsymbol{\lambda} \in \mathbb{R}^{N}$ and define

$$
\phi=\sum_{i=1}^{N} \lambda_{i} \mathrm{G}^{i} .
$$

The function $\phi$ is periodic and belongs to the space $H^{1}\left(Y_{t}\right)$. We prove that $\mathbf{K}$ is semi-positive definite:

$$
\left|Y_{t}\right| \boldsymbol{\lambda}^{T} \mathbf{K} \boldsymbol{\lambda}=\int_{Y_{t}}\left|\nabla_{\mathbf{y}}(\phi-\mathbf{y} \cdot \boldsymbol{\lambda})\right|^{2} d \mathbf{y} \geq 0
$$

that is true for any $\nabla_{\mathbf{y}}(\phi-\mathbf{y} \cdot \boldsymbol{\lambda})$. The equality holds if and only if

$$
\nabla_{\mathbf{y}} \phi=\lambda
$$

However, under the assumption of periodicity in a connected domain, $\nabla_{\mathbf{y}} \phi=\boldsymbol{\lambda}$ if and only if $\nabla_{\mathbf{y}} \phi=$ $\boldsymbol{\lambda}=\mathbf{0}$. Therefore, $\mathbf{K}$ is positive definite.

Remark 3.2. The interstitial domain $Y_{t}$ has to be connected to guarantee the positive definiteness of the tensor $\boldsymbol{K}$ (otherwise, it is semi-positive definite).

Lemma 3.3. If the capillary domain $Y_{c}$ is connected, then the tensor $\boldsymbol{E}$ is symmetric and positive definite.

Proof. We proceed analogously as [40]. Thanks to the Lax-Milgram lemma, there exist a unique weak solution to problem (8), which variational formulation reads as

$$
\int_{Y_{c}} \nu \nabla_{\mathbf{y}} \mathbf{W}^{j}: \nabla_{\mathbf{y}} \mathbf{v} d \mathbf{y}-\int_{Y_{c}} \mathbf{e}_{j} \cdot \mathbf{v} d \mathbf{y}=0
$$


for any periodic $\mathbf{v} \in H^{1}\left(Y_{c}\right)$ such that $\nabla_{\mathbf{y}} \cdot \mathbf{v}=0$ and $\mathbf{v} \cdot \mathbf{n}=0$ on $\Gamma_{Y}$. Taking $\mathbf{v}=\mathbf{W}^{i}$ the following identity holds:

$$
\begin{aligned}
\left|Y_{c}\right|[\mathbf{E}]_{i j} & =\int_{Y_{c}} \mathbf{W}^{j} \cdot \mathbf{e}_{i} d \mathbf{y} \\
& =\int_{Y_{c}} \nu \nabla_{\mathbf{y}} \mathbf{W}^{j}: \nabla_{\mathbf{y}} \mathbf{W}^{i} d \mathbf{y} .
\end{aligned}
$$

Therefore the tensor is symmetric. To prove that it is positive definite, we take any $\boldsymbol{\lambda} \in \mathbb{R}^{N}$ and define

$$
\boldsymbol{\psi}=\sum_{i=1}^{N} \lambda_{i} \mathbf{W}^{i}
$$

We first prove that $\boldsymbol{\lambda}^{T} \mathbf{E} \boldsymbol{\lambda}$ is non-negative. Indeed,

$$
\left|Y_{c}\right| \boldsymbol{\lambda}^{T} \mathbf{E} \boldsymbol{\lambda}=\int_{Y_{c}} \nu \nabla_{\mathbf{y}} \psi: \nabla_{\mathbf{y}} \psi d \mathbf{y} \geq 0
$$

The equality holds if and only if $\nabla_{\mathbf{y}} \psi=0$. Then, the following equation must be satisfied

$0=\int_{Y_{c}} \nu \nabla_{\mathbf{y}} \boldsymbol{\psi}: \nabla_{\mathbf{y}} \mathbf{v} d \mathbf{y}-\int_{\Gamma_{Y}}\left(\left[\left(\mathbf{n} \cdot \nabla_{\mathbf{y}}\right) \boldsymbol{\psi}\right] \cdot \mathbf{n}\right)(\mathbf{v} \cdot \mathbf{n}) d s=\int_{Y_{c}} \boldsymbol{\lambda} \cdot \mathbf{v} d \mathbf{y}, \quad \forall \mathbf{v} \in H^{1}\left(Y_{c}\right): \nabla_{\mathbf{y}} \cdot \mathbf{v}=0$

Since (10) holds for any $\mathbf{v}$ in the appropriate space defined above, it is valid also for $\mathbf{v}=\boldsymbol{\lambda}$. Therefore, we conclude that (10) is true if and only if $\boldsymbol{\lambda}=\mathbf{0}$ and state that $\mathbf{E}$ is positive definite.

Remark 3.4. When the domain $Y_{c}$ is not connected, then the unique solution to problem (8) is $\boldsymbol{W}^{j}=\boldsymbol{O}$ and $P^{j}=y_{j}$. In this case the tensor $\boldsymbol{E}$ is zero.

Remark 3.5. The tensor $\bar{\kappa} \boldsymbol{K}+\frac{\left|Y_{c}\right|}{\left|Y_{t}\right|} \boldsymbol{E}$ is symmetric and positive definite since it is the sum of two symmetric and positive definite tensors.

Remark 3.6. If one of the two domains $\left(\Omega_{c}^{\varepsilon}\right.$ or $\left.\Omega_{t}^{\varepsilon}\right)$ is not connected, then $p_{c}=p_{t}$.

\subsection{Limit problems}

In this section, we show the link between the different models. We assume that both instertitium and capillary phases are connected so that the 3 models complemented with Dirichlet, Neumann or Robin conditions are well-posed. 


\section{Equivalence of model 2 and 3}

First consider model (2) and (3) with Dirichlet conditions. Assuming that both $\bar{R}_{\mathbf{n}}$ and $\kappa$ are of order of magnitude $\varepsilon$, model (3) reads as follows:

$$
\begin{array}{cc}
\varepsilon \nabla \cdot\left(\bar{\kappa} \mathbf{K} \nabla p_{t}\right)=\varepsilon \frac{\bar{R}_{\mathbf{n}}\left|\Gamma_{Y}\right|}{\left|Y_{t}\right|}\left(p_{t}-p_{c}\right) & \text { in } \Omega, \\
\nabla \cdot\left(\mathbf{E} \nabla p_{c}\right)=\varepsilon \frac{\bar{R}_{\mathbf{n}}\left|\Gamma_{Y}\right|}{\left|Y_{c}\right|}\left(p_{c}-p_{t}\right) & \text { in } \Omega, \\
\left.p_{t}\right|_{\partial \Omega}=p_{t, \infty},\left.\quad p_{c}\right|_{\partial \Omega}=p_{c, \infty}, & \text { on } \partial \Omega .
\end{array}
$$

It is clear that $(11 b)$ is not a singluar perturbation of the operator $\nabla \cdot(\mathbf{E} \nabla \cdot)$ in the sense of Kato [42] and thus the solution to problem (11) tends to the solution to the following problem, which is nothing that model (3):

$$
\begin{array}{cl}
\nabla \cdot\left(\bar{\kappa} \mathbf{K} \nabla p_{t}\right)=\frac{\bar{R}_{\mathbf{n}}\left|\Gamma_{Y}\right|}{\left|Y_{t}\right|}\left(p_{t}-p_{c}\right) & \text { in } \Omega, \\
\nabla \cdot\left(\mathbf{E} \nabla p_{c}\right)=0 & \text { in } \Omega, \\
\left.p_{t}\right|_{\partial \Omega}=p_{t, \infty},\left.\quad p_{c}\right|_{\partial \Omega}=p_{c, \infty}, & \text { on } \partial \Omega .
\end{array}
$$

\section{Equivalence of model 1 and 2}

Considering $\bar{R}_{\mathbf{n}}$ of the order of $\varepsilon^{-1}$ and $\bar{\kappa}$ of the order of 1 , model (2) of Table 1 reads then

$$
\begin{array}{cc}
\varepsilon \nabla \cdot\left(\bar{\kappa} \mathbf{K} \nabla p_{t}\right)=\frac{\bar{R}_{\mathbf{n}}\left|\Gamma_{Y}\right|}{\left|Y_{t}\right|}\left(p_{t}-p_{c}\right) & \text { in } \Omega, \\
\varepsilon \nabla \cdot\left(\mathbf{E} \nabla p_{c}\right)=\frac{\bar{R}_{\mathbf{n}}\left|\Gamma_{Y}\right|}{\left|Y_{c}\right|}\left(p_{c}-p_{t}\right) & \text { in } \Omega \\
\left.p_{t}\right|_{\partial \Omega}=p_{t, \infty},\left.\quad p_{c}\right|_{\partial \Omega}=p_{c, \infty}, & \text { on } \partial \Omega .
\end{array}
$$

Here the asymptotic analysis is much trickier since both equations (12a)-(12b) are singular perturbation of the div-grad operator. In particular, a delicate asymptotic analysis makes appear a exponential decay of the $p_{t}-p_{c}$ from the boundary, showing that out of the vicinity of the tumor boundary, both pressures are equal. The details of this results are given in [43], however we expose here the main arguments in the simple case where $\bar{\kappa} \mathbf{K}$ and $\mathbf{E}$ are colinear to the identity, that is for a $\lambda \neq 0$ :

$$
\bar{\kappa} \mathbf{K}=\lambda \mathbf{E}
$$

Then simple calculation shows that

$$
\nabla \cdot\left(\mathbf{E} \nabla\left(p_{t}-p_{c}\right)\right)=\frac{\bar{R}_{\mathbf{n}}}{\varepsilon}\left(\lambda \frac{\left|\Gamma_{Y}\right|}{\left|Y_{t}\right|}+\frac{\left|\Gamma_{Y}\right|}{\left|Y_{c}\right|}\right)\left(p_{t}-p_{c}\right) \quad \text { in } \Omega
$$


It is well-known, especially in conduction theory [44, 45] that problem (13) makes appear a so-called skin depth effect: the pressure difference $p_{t}-p_{c}$ decays exponentially fast from the boundary. More precisely, denoting by $\alpha$ the factor given by

$$
\alpha=\sqrt{\bar{R}_{\mathbf{n}}\left(\lambda \frac{\left|\Gamma_{Y}\right|}{\left|Y_{t}\right|}+\frac{\left|\Gamma_{Y}\right|}{\left|Y_{c}\right|}\right)}
$$

hence in the local coordinates near the boundary

$$
p_{t}-p_{c}=\left(p_{t, \infty}-p_{c, \infty}\right) e^{-\frac{\alpha}{\sqrt{\varepsilon}} x_{n}}+o(\varepsilon)
$$

where $x_{n}$ is the normal variable with respect to the tumor boundary.

Interestingly, we thus obtain that in this asymptotic regime, the solution to model (2) with Dirichlet boundary conditions can be approached by the solution to model (1) with the following appropriate boundary condition

$$
\begin{array}{ll}
\nabla \cdot((\lambda+1) \mathbf{E} \nabla p)=0, & \text { in } \Omega \\
\left.p\right|_{\partial \Omega}=\frac{1}{2}\left(p_{t, \infty}+p_{c, \infty}\right), & \text { on } \partial \Omega .
\end{array}
$$

The rogorous proof is given in [43]. The result involves Riemannian geometry results which are far from the scope of this paper, however the general idea of the exponential decay of the pressure difference remains.

\section{Numerical simulations}

The Galerkin Finite Elements Method was used to discretize the equations in order to test the homogenized model in equation (2). 3D simulations were run in order to analyse the impact of the micro-scale geometry on the homogenized solutions and the influence of the vessel permeability $R_{\mathbf{n}}$ on the fluid transport. Regarding the homogenized models, the following strategy has been adopted:

- The periodic cell was considered as the unit cube $(0,1)^{3}$ in $\mathbb{R}^{3}$. The domain was divided in two regions $\left(Y_{t}\right.$ and $\left.Y_{c}\right)$ and the software Gmsh was used to perform the triangulation $\mathcal{T}_{h}$. Problem (8) was discretized with the Galerkin Finite Elements Method. Piecewise linear polynomials $\left(\mathbb{P}_{1}\right)$ were used for the variable $\mathrm{P}^{j}$. For the variable $\mathbf{W}^{j}$, we used piecewise linear polynomials with bubbles $\left(\mathbb{P}_{1 b}=\left\{v \in H^{1}(\Omega):\left.\forall K \in \mathcal{T}_{h} \quad v\right|_{K} \in \mathbb{P}_{1} \oplus \operatorname{Span}\left\{\lambda_{0}^{K} \lambda_{1}^{K} \lambda_{2}^{K} \lambda_{3}^{K}\right\}\right\}\right.$, where $\lambda_{j}^{K}, j=0, \ldots, N$ are the 4 barycentric coordinate functions of the element $K$ ). Problem (7) was solved on the domain $Y_{t}$ using piecewise linear polynomials $\left(\mathbb{P}_{1}\right)$ for the variable $\mathrm{G}^{j}$.

- The tensors $\mathbf{K}$ and $\mathbf{E}$ were computed according to (9).

- The homogenized model was simulated on the normalized sphere of radius 0.5. Models in Table 1 were simulated using the Galerkin Finite Elements Method. Quadratic piecewise elements $\left(\mathbb{P}_{2}\right)$ were used for both $p_{t}$ and $p_{c}$. 

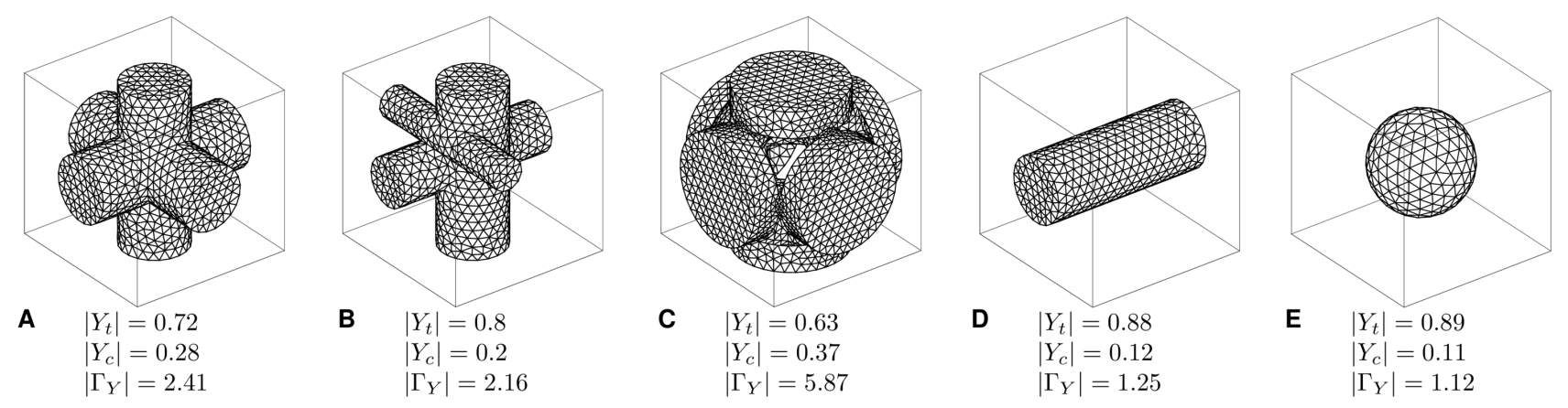

Figure 3. Different structures of the unit periodic cell with the respective volume and surface fractions. The mesh represents the capillary domain $Y_{c}$, while the difference between the box and the mesh is the interstitial compartment $Y_{t}$.

\begin{tabular}{cccccccccc}
\hline & $\mathbf{K}_{11}$ & $\mathbf{K}_{12}$ & $\mathbf{K}_{13}$ & $\mathbf{K}_{21}$ & $\mathbf{K}_{22}$ & $\mathbf{K}_{23}$ & $\mathbf{K}_{31}$ & $\mathbf{K}_{32}$ & $\mathbf{K}_{33}$ \\
\hline Fig 3a & 0.808 & $7.5 \mathrm{e}-5$ & $7.89 \mathrm{e}-6$ & $7.5 \mathrm{e}-5$ & 0.808 & $5.49 \mathrm{e}-5$ & $7.89 \mathrm{e}-6$ & $5.49 \mathrm{e}-5$ & 0.808 \\
Fig 3b & 0.877 & $-1.76 \mathrm{e}-3$ & $3.91 \mathrm{e}-3$ & $-1.76 \mathrm{e}-3$ & 0.814 & $2.29 \mathrm{e}-3$ & $3.91 \mathrm{e}-3$ & $2.29 \mathrm{e}-3$ & 0.933 \\
Fig 3c & 0.72 & $-1.09 \mathrm{e}-4$ & $1.03 \mathrm{e}-4$ & $-1.09 \mathrm{e}-4$ & 0.72 & $3.98 \mathrm{e}-5$ & $1.03 \mathrm{e}-4$ & $3.98 \mathrm{e}-5$ & 0.72 \\
Fig 3d & 1 & $-3.19 \mathrm{e}-8$ & $-9.81 \mathrm{e}-8$ & $-3.19 \mathrm{e}-8$ & 0.895 & $1.01 \mathrm{e}-4$ & $-9.81 \mathrm{e}-8$ & $1.01 \mathrm{e}-4$ & 0.895 \\
Fig 3e & 0.954 & $-4.69 \mathrm{e}-5$ & $5.2 \mathrm{e}-5$ & $-4.69 \mathrm{e}-5$ & 0.954 & $8.14 \mathrm{e}-5$ & $5.2 \mathrm{e}-5$ & $8.14 \mathrm{e}-5$ & 0.954 \\
\hline \hline & $\mathbf{E}_{11}$ & $\mathbf{E}_{12}$ & $\mathbf{E}_{13}$ & $\mathbf{E}_{21}$ & $\mathbf{E}_{22}$ & $\mathbf{E}_{23}$ & $\mathbf{E}_{31}$ & $\mathbf{E}_{32}$ & $\mathbf{E}_{33}$ \\
\hline Fig 3a & $2.2 \mathrm{e}-3$ & $8 \mathrm{e}-6$ & $-1.1 \mathrm{e}-6$ & $8 \mathrm{e}-6$ & $2.2 \mathrm{e}-3$ & $-1.3 \mathrm{e}-5$ & $-1.1 \mathrm{e}-6$ & $-1.3 \mathrm{e}-5$ & $2.2 \mathrm{e}-3$ \\
Fig 3b & $9.5 \mathrm{e}-4$ & $8.1 \mathrm{e}-7$ & $2.3 \mathrm{e}-5$ & $8.1 \mathrm{e}-7$ & $1.8 \mathrm{e}-4$ & $7.7 \mathrm{e}-6$ & $2.3 \mathrm{e}-5$ & $7.7 \mathrm{e}-6$ & $2.9 \mathrm{e}-3$ \\
Fig 3c & $4.0 \mathrm{e}-4$ & $-8.7 \mathrm{e}-7$ & $-7.4 \mathrm{e}-7$ & $-8.7 \mathrm{e}-7$ & $4.0 \mathrm{e}-4$ & $-2.7 \mathrm{e}-6$ & $-7.4 \mathrm{e}-7$ & $-2.7 \mathrm{e}-6$ & $4.0 \mathrm{e}-4$ \\
Fig 3d & $4.7 \mathrm{e}-3$ & 0 & 0 & 0 & 0 & 0 & 0 & 0 & 0 \\
Fig 3e & 0 & 0 & 0 & 0 & 0 & 0 & 0 & 0 & 0 \\
\hline
\end{tabular}

Table 2. Values of the tensors $\mathbf{K}$ and $\mathbf{E}$ for the different microstructures depicted in Fig. 3.

\subsection{Cell problems: tensor properties varying the microstructure}

The tensors $\mathbf{K}$ and $\mathbf{E}$ defined in (9) have different properties according to the microstructure. To analyse them, we solved equations (7) and (8) in the unitary cell, i.e. the cube $(0,1)^{3} \subset \mathbb{R}^{3}$. Different geometric configurations for the domains $Y_{t}$ and $Y_{c}$ were tested (Figure 3).

Table 2 provides the values of the elements in the two tensors $\mathbf{K}$ and $\mathbf{E}$. These results confirm the analysis done in Section 3.1. Indeed, the tensors $\mathbf{K}$ and $\mathbf{E}$ are symmetric and positive definite when the two domains are connected (Fig. 3a, 3b and 3c). When the capillaries are not connected in all the directions (Fig. 3d, 3e), the tensor $\mathbf{E}$ is semi-positive definite as the solution to the cell problems (7) is trivial: $\mathbf{W}^{j}=0$ and $\mathrm{P}^{j}=\mathbf{e}_{j}, j=1,2,3$. Figure 3 provides the values of the interstitial and capillary volume fractions $\left(\left|Y_{t}\right|\right.$ and $\left|Y_{c}\right|$, respectively) and of the vascular surface $\Gamma_{Y}$. 


\subsection{Macroscopic dynamic of fluid transport in tumours}

We eventually considered realistic parameters to test model (2). The homogenized model was tested with a tumor considered as a sphere of normalized radius 0.5. Table 3 provides the values of the parameters of the model. Regarding the interstitial hydraulic conductivity $k_{t}$, the vascular permeability $L_{p}$ and the tumour characteristic length $L$, we considered values relative to different tissues, as summarized in Tables 4, 5 and 6 , respectively. Simulations were run considering different microstructures, namely the ones shown in Figs 3a-c. Dirichlet boundary conditions were considered for the interstitial and capillary pressure, specifically $p_{t, \infty}=0$ and $p_{c, \infty}=1$ (normalized values).

\section{Parameter influence}

First, we looked at the behaviour of the solution varying the parameters $k_{t}, L_{p}$ and $L$. Examples of solutions as a function of the radius are shown in Fig. 4. In this case, we considered the microstructure of Fig. 3c. Results relative to the interstitial pressure and velocity were in agreement with the ones found in [2], where the authors considered the following model:

$$
\nabla \cdot\left(\mathbf{K} \nabla p_{t}\right)=\frac{\bar{R}_{\mathbf{n}} S}{\bar{\kappa} V}\left(p_{t}-p_{c}\right)
$$

where the vascular pressure $p_{c}$ is assumed to be constant and $S / V$ is the vascular area per unit volume of the tumour. Therefore, we considered this value to be equal to $\left|\Gamma_{Y}\right|$. The slight differences between the results obtained from the homogenized model and Baxter and Jain model (14) (Figure 4A) are due to the different rescaling of the equation, since we considered $S / V$ to be the vascular area per unit volume of the interstitial compartment $\left(\left|\Gamma_{Y}\right| /\left|Y_{t}\right|\right)$.

The interstitial fluid pressure is large and almost constant in the centre of the tumour and has a sharp drop at the periphery for increasing values of $\bar{R}_{\mathbf{n}}$ and decrasing values of $\bar{\kappa}$. As a consequence, the interstitial fluid velocity is almost zero in the centre of the tumour (since the pressure gradient is close to zero) and large at the periphery. The microvessel fluid pressure is almost constant and close to the value at the boundary. For large values of the parameter $\bar{R}_{\mathbf{n}}$, the capillary pressure decreases and gets closer to the interstitial fluid pressure. As a consequence, also the microvessel fluid velocity is close to zero in the centre of the tumour.

Eventually, we observed the skin depth effect of $p_{c}-p_{t}$ when the permeability of the vessel walls increases (Fig 4B). Indeed, the pressure difference is almost zero at the centre of the tumour and increases exponentially in correspondence of the boundary.

\section{Microstructure}

We fixed the parameter values $k_{t}=1.8 \cdot 10^{-12} \mathrm{~m}^{3} \cdot \mathrm{s} \cdot \mathrm{kg}^{-1}, L_{p}=1.86 \cdot 10^{-10} \mathrm{~m}^{2} \cdot \mathrm{s} \cdot \mathrm{kg}^{-1}$ and $L=5 \mathrm{~mm}$ and looked at the behaviour of the solutions relative to the different microstructures. Fig. 5 shows the results relative to the unitary cells of Fig 3A-C. In all cases, the IFP shows a sharp drop at the periphery and it equates the capillary pressure in the centre of the tumour, while the capillary pressure is approximately constant in the whole tumour. The interstitial fluid velocity $\mathbf{u}_{t}$ is directed 


\begin{tabular}{lllll}
\hline Parameter & Description & Value & Unit & Reference \\
\hline$\mu$ & blood viscosity & $4 \cdot 10^{-3}$ & $\mathrm{~kg} \cdot \mathrm{m}^{-1} \cdot \mathrm{s}^{-1}$ & {$[49]$} \\
$d$ & mean intercapillary distance & $50 \cdot 10^{-6}$ & $\mathrm{~m}$ & {$[50]$} \\
$\alpha_{\mathrm{BJ}}$ & BJS constant & 1 & - & - \\
$p_{t, \infty}$ & surrounding interstitial pressure & 0 & $\mathrm{mmHg}$ & - \\
$p_{c, \infty}$ & surrounding capillary pressure & {$[15,80]$} & $\mathrm{mmHg}$ & {$[7]$} \\
\hline
\end{tabular}

Table 3. Fixed parameters used to simulate IFP and IFV.

\begin{tabular}{lll}
\hline Tissue & $k_{t}\left[\mathrm{~m}^{3} \cdot \mathrm{s} \cdot \mathrm{kg}^{-1}\right]$ & Reference \\
\hline Dog squamous cell tissue & $1.8 \cdot 10^{-12}$ & {$[51]$} \\
Mouse mammary carcinoma & $1.88 \cdot 10^{-13}$ & {$[52]$} \\
Hepatoma 5123 in vivo & $2.9 \cdot 10^{-15}$ & {$[53]$} \\
\hline
\end{tabular}

Table 4. Values of the interstitial hydraulic conductivity $k_{t}$ of different tissues.

outward from the domain, while the blood velocity is directed inward. The two velocities are radially homogeneous in cases $5 \mathrm{~A}$ and $5 \mathrm{C}$, while they show asymmetries in case $5 \mathrm{~B}$ due to the asymmetric microscopic structure of Fig 3B.

We noticed that only when the capillary subdomain is smaller than the interstitial region, the blood velocity is larger than the interstitial fluid flow (data not shown). This is biologically relevant as the capillary volume fraction is usually within the range $[16 \%, 50 \%][46]$ and the average blood velocity is larger than the interstitial fluid velocity $[47,48]$.

\section{Boundary conditions}

Eventually, we tested model (2) with different boundary conditions. In particular, Neumann boundary conditions were considered for the capillary pressure, in order to ensure the continuity of the normal velocity in the vessels at the tumour periphery:

$$
-\mathbf{E} \nabla p_{c} \cdot \mathbf{n}=\mathbf{u}_{c, \infty} \cdot \mathbf{n},
$$

where $\mathbf{u}_{c, \infty}$ is the blood velocity in the sourranding tissue. Dirichlet boundary conditions were imposed to the interstitial pressure. Well-posedness of model (2) is guaranteed with this set of boundary conditions for $\left(p_{t}, p_{c}\right) \in H_{0}^{1}(\Omega) \times H^{1}(\Omega)$.

We ran experiments with different boundary conditions for the capillary pressure $p_{c}$ as summarized in Table 7. Homogeneous Dirichlet boundary conditions were considered for the interstitial fluid pressure $p_{t}$. Figure S1 shows the results at the centre of the sphere as function of the normalized radius. The interstitial pressure increases at the centre of the tumour and equates the blood pressure in the three cases. When considering the case "Neumann 2 ", the blood velocity is constantly high inside the domain and the capillary pressure profile is therefore due to the gradient along the $x$-axis. 


\begin{tabular}{lll}
\hline Tissue & $L_{p}\left[\mathrm{~m}^{2} \cdot \mathrm{s} \cdot \mathrm{kg}^{-1}\right]$ & Reference \\
\hline Mouse mammary carcinoma & $1.86 \cdot 10^{-10}$ & {$[52]$} \\
R3230 mammary adenocarcinoma & $4.5 \cdot 10^{-11}$ & {$[54]$} \\
Healty rat hindquarter tissue & $2.3 \cdot 10^{-12}$ & {$[55]$} \\
\hline
\end{tabular}

Table 5. Values of the vessel permeability $L_{p}$ of different tissues.

\begin{tabular}{lll}
\hline Characteristic length $L[\mathrm{~mm}]$ & Tumor volume $\left[\mathrm{mm}^{3}\right]$ & $\varepsilon=d / L$ \\
\hline 5 & 4.2 & 0.05 \\
10 & 523.6 & 0.01 \\
15 & 4200 & 0.005 \\
\hline
\end{tabular}

Table 6. Characteristic length (diameter) of the tumour and corresponding tumour volume and value of $\varepsilon$.

A
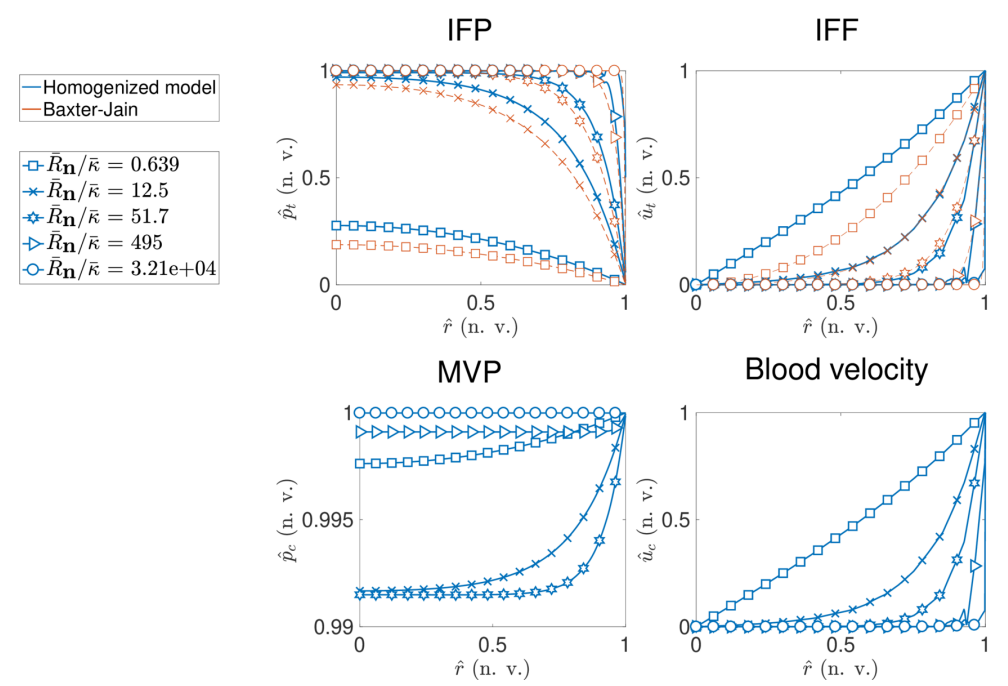

B

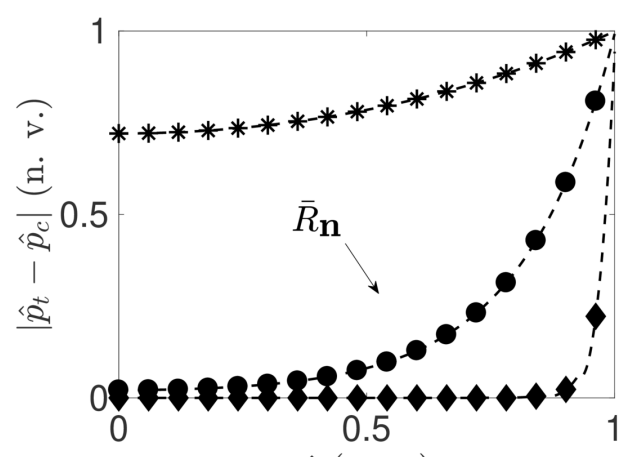

$\hat{r}$ (n. v.)

Figure 4. (A) Normalized values (n.v.) of interstitial fluid pressure and flow (IFP and IFF), of microvascular pressure (MVP) and of blood velocity as functions of the normalized radius $\hat{r}$ varying the parameter $\bar{R}_{\mathbf{n}} / \kappa$. The microstructure considered in this case corresponds to Fig 3c. The blue lines are the simulations of the homogenized model (2) and the red lines are the results of Baxter and Jain model [2]. (B) Difference between $\hat{p}_{c}$ and $\hat{p}_{t}$ in normalized values (n.v.) as functions of the normalized radius $\hat{r}$ varying the parameter $\bar{R}_{\mathbf{n}}$ and with $\bar{\kappa}$ fixed.

\begin{tabular}{lll}
\hline Experiment & Boundary condition $($ on $\partial \Omega)$ & Parameter value (normalized) \\
\hline Dirichlet & $p_{c}=p_{c, \infty}$ & $p_{c, \infty}=1$ \\
Neumann 1 & $-\mathbf{E} \nabla p_{c} \cdot \mathbf{n}=\mathbf{u}_{c, \infty} \cdot \mathbf{n}$ & $\mathbf{u}_{c, \infty}=-1 \cdot 10^{-3} \mathbf{n}$ \\
Neumann 2 & $-\mathbf{E} \nabla p_{c} \cdot \mathbf{n}=\mathbf{u}_{c, \infty} \cdot \mathbf{n}$ & $\mathbf{u}_{c, \infty}=\left[-1 \cdot 10^{-5}, 0,0\right]^{T}$ \\
\hline
\end{tabular}

Table 7. Different boundary conditions considered for the microvessel pressure $p_{c}$. 


\section{IFP}

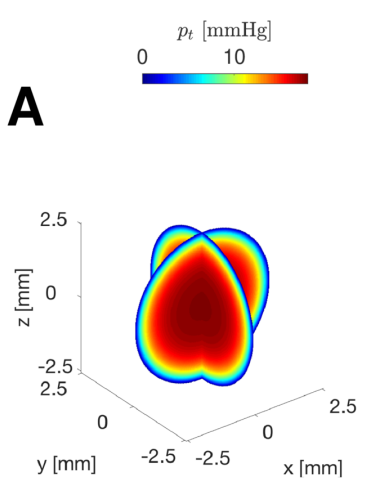

\section{MVP}

$\begin{array}{rr}p_{c}[\mathrm{mmHg}] \\ 17.9 & 18\end{array}$

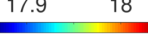

IFF

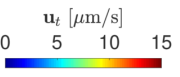

Blood velocity

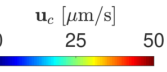

\section{B}
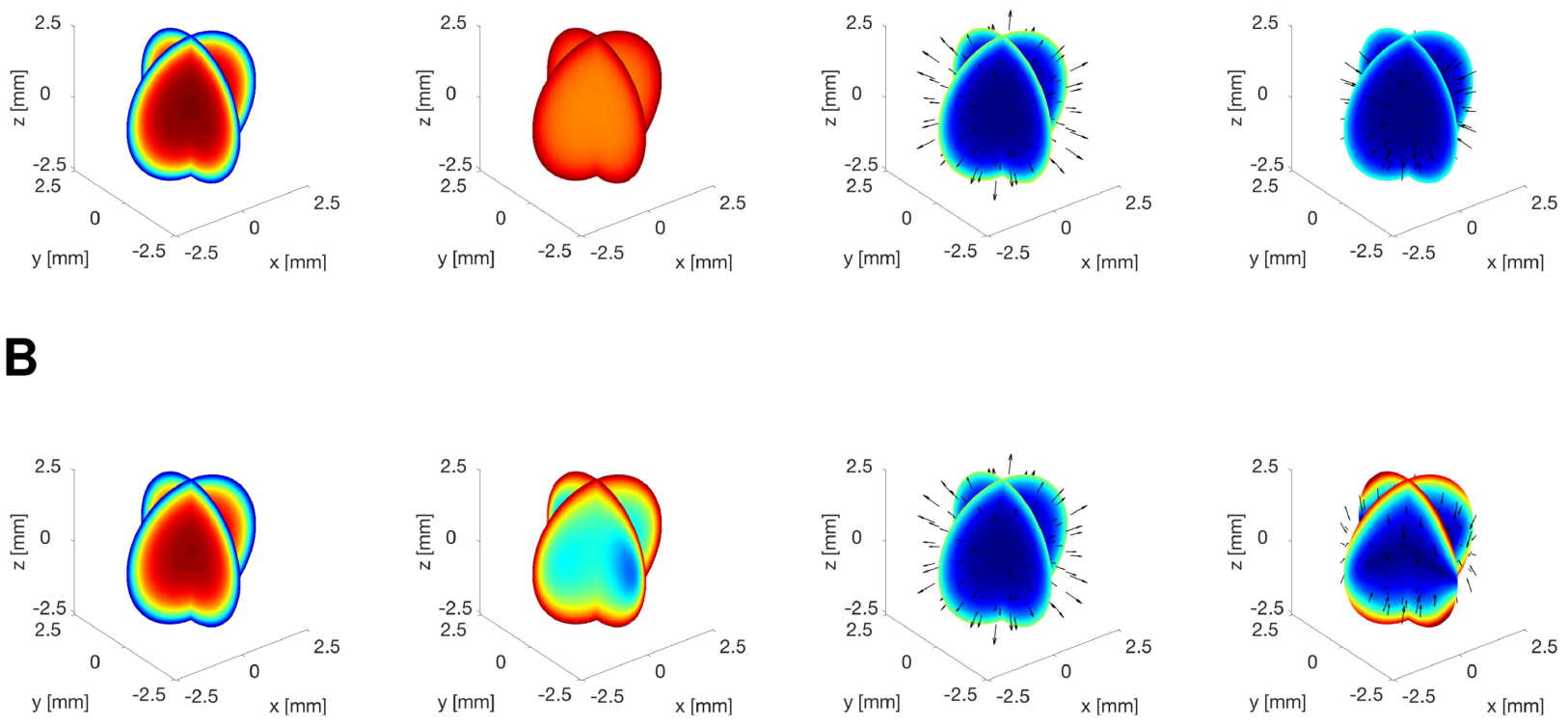

\section{C}
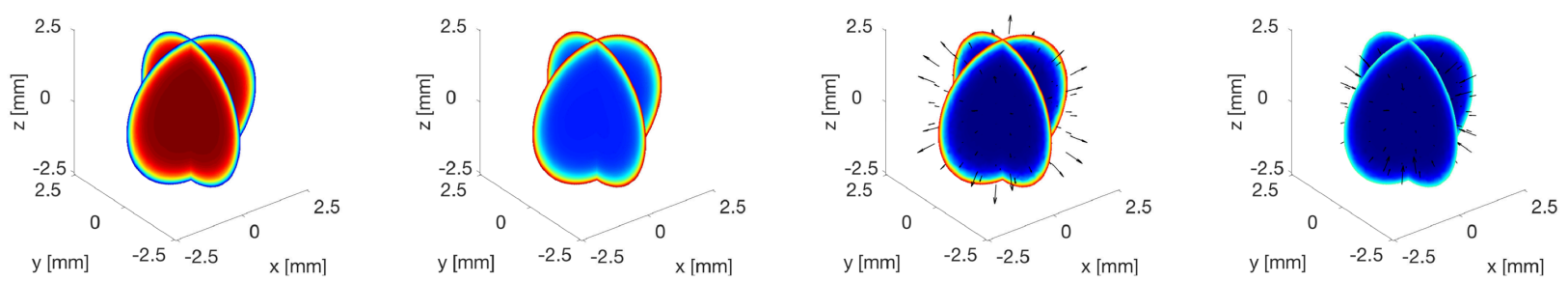

Figure 5. 3D slices at the centre of the sphere with the interstitial pressure (first column), the capillary pressure (second comumn), interstitial velocity (third column) and capillary velocity (fourth column). Results were computed using the microstructure of Fig 3a (A), of Fig 3b (B) and of Fig 3c (C) and setting $k_{t}=1.8 \cdot 10^{-12}$ $\mathrm{m}^{3} \cdot \mathrm{s} \cdot \mathrm{kg}^{-1}, L_{p}=1.86 \cdot 10^{-10} \mathrm{~m}^{2} \cdot \mathrm{s} \cdot \mathrm{kg}^{-1}$ and $L=5 \mathrm{~mm}$. IFP = interstitial fluid pressure, MVP $=$ microvascular pressure, IFF $=$ interstitial fluid flow. 


\section{Discussion}

We have provided an analysis of the impact of microstructure properties of the tumour employing the homogenisation theory.

First, we have described a model at the microscopic scale that couples vascular, transvascular and interstitial fluids, adopting an asymptotic expansion technique. Then, we have derived three macroscale models according to the vessel wall permeability and the interstitial hydraulic conductivity. After having analysed the well-posedness of the problems, we performed numerical simulations to assess some properties according to the microstructure.

Well-posedness is guaranteed when the two subdomains $Y_{t}$ and $Y_{c}$ are connected. When one region is not connected with respect to one axis, the fluid is not transported along this direction. For example, in Fig. 3e the capillary microstructure is a closed sphere, therefore there is no fluid transport in the blood vessels; in Fig. 3d, the vessel geometry is connected only along the $x$-axis that is the only direction for the capillary fluid flow. This represents a limit for the 2D simulations, as the subdomains $Y_{t}$ and $Y_{c}$ cannot be both connected. In this case, one among the interstitial or the vessel flow is always zero. However, tensors $\mathbf{K}$ and $\mathbf{E}$ can be determined by calibrating directly the homogenized models to medical imaging data.

Furthermore, we motivated the links between the various regimes and shown that model (2) covers a wide range of cases, confirming previous results [29]. In particular, we have shown that model (1) is equivalent to model (2) under certain conditions and that model (2) can be approximated to model (3) under certain assumptions on the parameters.

Eventually, we calibrated model (2) with parameters taken from the literature and analysed their influence on the solutions. We observed that different microstructures and different sets of boundary conditions strongly impact the macroscopic dynamics of the fluids. The geometric shape of the unitary cell influences the isotropy of the capillary fluid velocity, while the vascular volume fraction affects the blood velocity. Indeed, when the capillary volume fraction $\left|Y_{c}\right|$ is large, the blood velocity $\mathbf{u}_{c}$ is equal or lower than the interstitial fluid velocity $\mathbf{u}_{t}$. This might not be biologically relevant. On the other hand, when the capillary volume fraction is smaller the blood velocity is of higher magnitude and gets closer to the average values (around $1.62 \mathrm{~mm} \cdot \mathrm{s}^{-1}[56]$ ). This confirms that the homogenized models are consistent with biological observations. Indeed, the vascular volume fraction lies within the values of $16 \%$ and $50 \%$. [46, 57, 58]. Moreover, the average values of the pressures and of the velocities obtained from simulations with different sets of boundary conditions were compared against literature values. When Dirichlet-Dirichlet boundary conditions are considered, both the interstitial and the capillary pressures fit better the well-known profile of the IFP that is high at the centre of the tumour and shows a sharp drop at the periphery [2]. However, when Dirichlet-Neumann boundary conditions are considered for the interstitial and the capillary pressure, respectively, the blood velocity reaches average values closer to the literature ones. Possible improvements of our computations might be achieved by considering the correctors and by adding boundary layers, to take into account the Dirichlet boundary conditions that are imposed to the true solution $\left(p_{t}^{\varepsilon}, p_{c}^{\varepsilon}\right)$ of the micro-scale model, but are not satisfied by the periodic solutions to the homogenized ones.

The current work focuses on the analysis of asymptotic models that describe fluid transport in tumour tissues. Fluid velocities are necessary to develop convection-diffusion models for the description 
of drug transport in tumour tissues. This motivated our choice of a steady-state model, as in reality, the time variation of the fluid transport is negligible with respect to the evolution of drug distribution inside the tumour. However, spatial tumour growth might be included in the model.

Further extensions might include a relaxation of the periodicity hypothesis, that might not be realistic in a biological context, as tumours are highly heterogeneous. Assuming a random distribution of the capillaries, it is possible to define properly the representative volume element to better predict the fluid flow in the tumour [59]. Moreover, rheological effects of blood should be included to model blood transport in capillaries [60].

Applications of the models include the incorporation of 3D imaging data. Images provide the microstructure of the vessel network, that is necesssary to compute the correctors.

\section{References}

[1] Jain RK. Barriers to Drug Delivery in Solid Tumors. Sci Am. 1994 Jul;271(1):58-65.

[2] Baxter LT, Jain RK. Transport of Fluid and Macromolecules in Tumors. I. Role of Interstitial Pressure and Convection. Microvasc Res. 1989 Jan;37(1):77-104.

[3] Baish JW, Gazit Y, Berk DA, Nozue M, Baxter LT, Jain RK. Role of Tumor Vascular Architecture in Nutrient and Drug Delivery: An Invasion Percolation-Based Network Model. Microvasc Res. 1996 May;51(3):327-346.

[4] Chauhan VP, Stylianopoulos T, Boucher Y, Jain RK. Delivery of Molecular and Nanoscale Medicine to Tumors: Transport Barriers and Strategies. Annual Review of Chemical and Biomolecular Engineering. 2011 Jul;2(1):281-298.

[5] Helmlinger G, Netti PA, Lichtenbeld HC, Melder RJ, Jain RK. Solid Stress Inhibits the Growth of Multicellular Tumor Spheroids. Nat Biotechnol. 1997 Aug;15(8):778-783.

[6] Sevick EM, Jain RK. Viscous Resistance to Blood Flow in Solid Tumors: Effect of Hematocrit on Intratumor Blood Viscosity. Cancer Res. 1989 Jul;49(13):3513-3519.

[7] Boucher Y, Jain RK. Microvascular Pressure Is the Principal Driving Force for Interstitial Hypertension in Solid Tumors: Implications for Vascular Collapse. Cancer Res. 1992 Sep;52(18):51105114 .

[8] Netti PA, Berk DA, Swartz MA, Grodzinsky AJ, Jain RK. Role of Extracellular Matrix Assembly in Interstitial Transport in Solid Tumors. Cancer Res. 2000 May;60(9):2497-2503.

[9] Dvorak HF, Brown LF, Detmar M, Dvorak AM. Vascular Permeability Factor/Vascular Endothelial Growth Factor, Microvascular Hyperpermeability, and Angiogenesis. Am J Pathol. 1995 May;146(5):1029-1039.

[10] Young JS, Lumsden CE, Stalker AL. The Significance of the Tissue Pressure of Normal Testicular and of Neoplastic (Brown-Pearce Carcinoma) Tissue in the Rabbit. J Pathol Bacteriol. 1950 Jul;62(3):313-333. 
[11] Boucher Y, Baxter LT, Jain RK. Interstitial Pressure Gradients in Tissue-Isolated and Subcutaneous Tumors: Implications for Therapy. Cancer Res. 1990 Aug;50(15):4478-4484.

[12] Jain RK, Baxter LT. Mechanisms of Heterogeneous Distribution of Monoclonal Antibodies and Other Macromolecules in Tumors: Significance of Elevated Interstitial Pressure. Cancer Res. 1988 Dec;48(24 Pt 1):7022-7032.

[13] Jain RK. Transport of Molecules in the Tumor Interstitium: A Review. Cancer Res. 1987 Jun;47(12):3039-3051.

[14] Netti PA, Baxter LT, Boucher Y, Skalak R, Jain RK. Time-Dependent Behavior of Interstitial Fluid Pressure in Solid Tumors: Implications for Drug Delivery. Cancer Res. 1995 Nov;55(22):5451-5458.

[15] Starling EH. On the Absorption of Fluids from the Connective Tissue Spaces. J Physiol. 1896 May;19(4):312-326.

[16] Krogh A. The Anatomy and Physiology of Capillaries. New Haven, Yale Univ. Press; 1922.

[17] Apelblat A, Katzir-Katchalsky A, Silberberg A. A Mathematical Analysis of Capillary-Tissue Fluid Exchange. Biorheology. 1974 Feb;11(1):1-49.

[18] Blake TR, Gross JF. Analysis of Coupled Intra- and Extraluminal Flows for Single and Multiple Capillaries. Math Biosci. 1982 Jun;59(2):173-206.

[19] Baish JW, Netti PA, Jain RK. Transmural Coupling of Fluid Flow in Microcirculatory Network and Interstitium in Tumors. Microvasc Res. 1997 Mar;53(2):128-141.

[20] Pozrikidis C, Farrow DA. A Model of Fluid Flow in Solid Tumors. Annals of Biomedical Engineering. 2003 Feb;31(2):181-194.

[21] Soltani M, Chen P. Numerical Modeling of Fluid Flow in Solid Tumors. PLoS ONE. 2011 Jun;6(6):e20344.

[22] Bartha K, Rieger H. Vascular Network Remodeling via Vessel Cooption, Regression and Growth in Tumors. J Theor Biol. 2006 Aug;241(4):903-918.

[23] Welter M, Rieger H. Interstitial Fluid Flow and Drug Delivery in Vascularized Tumors: A Computational Model. PLoS ONE. 2013;8(8):e70395.

[24] Schuff MM, Gore JP, Nauman EA. A Mixture Theory Model of Fluid and Solute Transport in the Microvasculature of Normal and Malignant Tissues. I. Theory. J Math Biol. 2013 May;66(6):11791207.

[25] Owen MR, Alarcón T, Maini PK, Byrne HM. Angiogenesis and Vascular Remodelling in Normal and Cancerous Tissues. J Math Biol. 2009 Apr;58(4-5):689-721.

[26] Wijeratne PA, Hipwell JH, Hawkes DJ, Stylianopoulos T, Vavourakis V. Multiscale Biphasic Modelling of Peritumoural Collagen Microstructure: The Effect of Tumour Growth on Permeability and Fluid Flow. PLoS ONE. 2017 Sep;12(9):e0184511. 
[27] Zhao J, Salmon H, Sarntinoranont M. Effect of Heterogeneous Vasculature on Interstitial Transport within a Solid Tumor. Microvascular Research. 2007 May;73(3):224-236.

[28] Sweeney PW, d'Esposito A, Walker-Samuel S, Shipley RJ. Modelling the Transport of Fluid through Heterogeneous, Whole Tumours in Silico. PLoS Comput Biol. 2019 Jun;15(6):e1006751.

[29] Shipley RJ, Chapman SJ. Multiscale Modelling of Fluid and Drug Transport in Vascular Tumours. Bull Math Biol. 2010 Aug;72(6):1464-1491.

[30] Penta R, Ambrosi D, Quarteroni A. Multiscale Homogenization for Fluid and Drug Transport in Vascularized Malignant Tissues. Math Models Methods Appl Sci. 2015 Jan;25(01):79-108.

[31] Penta R, Ambrosi D, Shipley RJ. Effective Governing Equations for Poroelastic Growing Media. Q J Mech Appl Math. 2014 Feb;67(1):69-91.

[32] Penta R, Merodio J. Homogenized Modeling for Vascularized Poroelastic Materials. Meccanica. 2017 Nov;52(14):3321-3343.

[33] Shipley RJ, Sweeney PW, Chapman SJ, Roose T. A Four-compartment Multiscale Model of Fluid and Drug Distribution in Vascular Tumours. International Journal for Numerical Methods in Biomedical Engineering. 2020 Feb;

[34] Amar M, Andreucci D, Bisegna P, Gianni R. On a Hierarchy of Models for Electrical Conduction in Biological Tissues. Math Meth Appl Sci. 2006 May;29(7):767-787.

[35] Perrussel R, Poignard C. Asymptotic Expansion of Steady-State Potential in a High Contrast Medium with a Thin Resistive Layer. Appl Math Comput. 2013 Sep;221:48-65.

[36] Allaire G. Homogenization and Two-Scale Convergence. SIAM J Math Anal. 1992 Nov;23(6):14821518.

[37] Beavers GS, Joseph DD. Boundary Conditions at a Naturally Permeable Wall. J Fluid Mech. 1967 Oct;30(1):197-207.

[38] Voutouri C, Stylianopoulos T. Evolution of Osmotic Pressure in Solid Tumors. J Biomech. 2014 Nov;47(14):3441-3447.

[39] Saffman PG. On the Boundary Condition at the Surface of a Porous Medium. Stud Appl Math. 1971 Jun;50(2):93-101.

[40] Arbogast T, Lehr HL. Homogenization of a Darcy-Stokes System Modeling Vuggy Porous Media. Comput Geosci. 2006 Sep;10(3):291-302.

[41] Conca C. On the Application of the Homogenization Theory to a Class of Problems Arising in Fluid Mechanics. J Math Pures Appl. 1985;64:31-75.

[42] Kato T. Perturbation Theory for Linear Operators. vol. 132 of Classics in Mathematics. Berlin, Heidelberg: Springer Berlin Heidelberg; 1995. 
[43] Vaghi C, Benzekry S, Poignard C. Asymptotic Analysis of a Tumor Fluid Flow Model. To be submitted;

[44] Balanis CA. Advanced Engineering Electromagnetics. 2nd ed. Hoboken, N.J: John Wiley \& Sons; 2012.

[45] Haddar H, Joly P, Nguyen HM. Generalized Impedance Impedance Boundary Conditions For Scattering by Strongly Absorbing Obstacles: The Scalar Case. Math Models Methods Appl Sci. 2005 Aug;15(08):1273-1300.

[46] Forster JC, Harriss-Phillips WM, Douglass MJ, Bezak E. A Review of the Development of Tumor Vasculature and Its Effects on the Tumor Microenvironment. Hypoxia (Auckl). 2017;5:21-32.

[47] Walker-Samuel S, Roberts TA, Ramasawmy R, Burrell JS, Johnson SP, Siow BM, et al. Investigating Low-Velocity Fluid Flow in Tumors with Convection-MRI. Cancer Research. 2018 Apr;78(7):1859-1872.

[48] Kamoun WS, Chae SS, Lacorre DA, Tyrrell JA, Mitre M, Gillissen MA, et al. Simultaneous Measurement of RBC Velocity, Flux, Hematocrit and Shear Rate in Vascular Networks. Nat Methods. 2010 Aug;7(8):655-660.

[49] Rand PW, Lacombe E, Hunt HE, Austin WH. Viscosity of Normal Human Blood under Normothermic and Hypothermic Conditions. J Appl Physiol. 1964 Jan;19(1):117-122.

[50] Less JR, Skalak TC, Sevick EM, Jain RK. Microvascular Architecture in a Mammary Carcinoma: Branching Patterns and Vessel Dimensions. Cancer Res. 1991 Jan;51(1):265-273.

[51] Guyton AC, Granger HJ, Taylor AE. Interstitial Fluid Pressure. Physiol Rev. 1971 Jul;51(3):527563.

[52] Jain RK, Tong RT, Munn LL. Effect of Vascular Normalization by Antiangiogenic Therapy on Interstitial Hypertension, Peritumor Edema, and Lymphatic Metastasis: Insights from a Mathematical Model. Cancer Res. 2007 Mar;67(6):2729-2735.

[53] Swabb EA, Wei J, Gullino PM. Diffusion and Convection in Normal and Neoplastic Tissues. Cancer Res. 1974 Oct;34(10):2814-2822.

[54] Sevick EM, Jain RK. Measurement of Capillary Filtration Coefficient in a Solid Tumor. Cancer Res. 1991 Feb;51(4):1352-1355.

[55] Rippe B, Kamiya A, Folkow B. Simultaneous Measurements of Capillary Diffusion and Filtration Exchange during Shifts in Filtration-Absorption and at Graded Alterations in the Capillary Permeability Surface Area Product (PS). Acta Physiol Scand. 1978 Nov;104(3):318-336.

[56] Stamatelos SK, Kim E, Pathak AP, Popel AS. A Bioimage Informatics Based Reconstruction of Breast Tumor Microvasculature with Computational Blood Flow Predictions. Microvascular Research. 2014 Jan;91:8-21.

[57] Hilmas DE, Gillette EL. Morphometric Analyses of the Microvasculature of Tumors during Growth and after X-Irradiation. Cancer. 1974 Jan;33(1):103-110. 
[58] Vogel AW. Intratumoral Vascular Changes With Increased Size of a Mammary Adenocarcinoma: New Method and Results. J Natl Cancer Inst. 1965 May;34:571-578.

[59] Kanit T, Forest S, Galliet I, Mounoury V, Jeulin D. Determination of the Size of the Representative Volume Element for Random Composites: Statistical and Numerical Approach. International Journal of Solids and Structures. 2003 Jun;40(13-14):3647-3679.

[60] Pries AR, Secomb TW, Gessner T, Sperandio MB, Gross JF, Gaehtgens P. Resistance to Blood Flow in Microvessels in Vivo. Circ Res. 1994 Nov;75(5):904-915. 


\section{Supplementary Material}

\section{S1: Derivation of the microscale model with asymptotic expansion}

At the microscale, the domain $\Omega \in \mathbb{R}^{N}$ (with $N=2,3$ ) is the medium that consists of the interstitium $\Omega_{t}$, the vessel wall $\Omega_{m}$ and the capillary region $\Omega_{c}$. The interface between the capillary and the vessel wall and the one between the interstitium and the vessel wall are denoted respectively by $\Gamma=\partial \Omega_{c} \cap \partial \Omega_{m}$ and $\Gamma_{\delta}=\partial \Omega_{t} \cap \partial \Omega_{m}$. Figure $1 \mathrm{~A}$ shows the section of a capillary in the surrounding interstitium. In the three regions, the fluid flow is assumed to be incompressible. In the interstitium and in the capillaries, the equations explained in Section 2 hold.

Similarly to the interstitium, the capillary walls - of thickness $\delta$ - are considered as a porous medium with hydraulic conductivity $k_{m}$, therefore the fluid flow velocity $\mathbf{u}_{m}$ and pressure $p_{m}$ in the capillary walls are described by

$$
\nabla \cdot \mathbf{u}_{m}=0, \quad \mathbf{u}_{m}=-k_{m} \nabla p_{m} \quad \text { in } \Omega_{m} .
$$

\section{Interface conditions}

At the two boundaries $\Gamma$ and $\Gamma_{\delta}$, we have to consider interface conditions in order to couple the different equations. We make the following choices, similarly to [1]:

1. Continuity of the normal velocity on both $\Gamma$ and $\Gamma_{\delta}$ :

$$
\begin{array}{ll}
\mathbf{u}_{c} \cdot \mathbf{n}=\mathbf{u}_{m} \cdot \mathbf{n} & \text { on } \Gamma, \\
\mathbf{u}_{t} \cdot \mathbf{n}=\mathbf{u}_{m} \cdot \mathbf{n} & \text { on } \Gamma_{\delta} .
\end{array}
$$

This condition guarantees the continuity of mass through the two interfaces and it is a natural choice since the fluid is assumed to be incompressible in the three regions.

2. Balance of the normal forces at the interfaces $\Gamma, \Gamma_{\delta}$ :

$$
\begin{aligned}
p_{c}-\mu\left[(\mathbf{n} \cdot \nabla) \mathbf{u}_{c}\right] \cdot \mathbf{n} & =p_{m} & & \text { on } \Gamma, \\
p_{t} & =p_{m} & & \text { on } \Gamma_{\delta} .
\end{aligned}
$$

Condition (S4) is due to the fact that the blood force in $\Omega_{c}$ acting on $\Gamma$ is equal to the normal component of the Cauchy stress vector [2], while the only force in $\Omega_{m}$ acting on the interface is the Darcy pressure $p_{m}$. Analogously, equation (S5) is motivated by the fact that the only forces acting on the interface $\Gamma_{\delta}$ are the Darcy's pressures $p_{m}$ and $p_{t}$ in the respective regions $\Omega_{m}$ and $\Omega_{t}$.

3. Beavers-Joseph-Saffmann condition on the tangential component of the capillary velocity at the boundary with a porous medium $\Gamma$ :

$$
\mathbf{u}_{c} \cdot \boldsymbol{\tau}=-\frac{\sqrt{k_{m} \mu}}{\alpha_{\mathrm{BJ}}}\left[(\mathbf{n} \cdot \nabla) \mathbf{u}_{c}\right] \cdot \boldsymbol{\tau} \quad \text { on } \Gamma,
$$

where $\alpha_{\mathrm{BJ}}$ is a constant depending on the properties of the interface. This condition comes from the experimental evidence shown by Beavers and Joseph [3] who observed that the slip velocity along $\Gamma$ was proportional to the shear stress along $\Gamma$. Equation of the form (S6) was derived by Saffmann using a statistical approach and the Brinkman approximation for non-homogeneous porous medium [4] . 


\section{Non-dimensionalization}

We perform a dimensional analysis in order to understand the relative amplitude of the different parameters involved. We rescale our fields as follows:

$$
\mathbf{x}=L \mathbf{x}^{\prime}, \quad \mathbf{u}=U \mathbf{u}^{\prime}, \quad p=\frac{\mu L U}{d^{2}} p^{\prime}+p_{0},
$$

where $L$ is the characteristic domain length, $d$ is the mean intercapillary distance and $U$ is a characteristic velocity. The non-dimensional fluid transport problem reads (neglecting the primes for the sake of simplicity)

$$
\begin{aligned}
& \nu \nabla^{2} \mathbf{u}_{c}=\nabla p_{c}, \quad \nabla \cdot \mathbf{u}_{c}=0, \quad \text { in } \Omega_{c}, \\
& \mathbf{u}_{t}=-\kappa \nabla p_{c}, \quad \nabla \cdot \mathbf{u}_{t}=0, \quad \text { in } \Omega_{t}, \\
& \mathbf{u}_{m}=-\kappa_{m} \nabla p_{m}, \quad \nabla \cdot \mathbf{u}_{m}=0, \quad \text { in } \Omega_{m}, \\
& \mathbf{u}_{c} \cdot \mathbf{n}=\mathbf{u}_{m} \cdot \mathbf{n} \quad \text { on } \Gamma \text {, } \\
& \mathbf{u}_{t} \cdot \mathbf{n}=\mathbf{u}_{m} \cdot \mathbf{n} \quad \text { on } \Gamma_{\delta}, \\
& p_{c}-\nu\left[(\mathbf{n} \cdot \nabla) \mathbf{u}_{c}\right] \cdot \mathbf{n}=p_{m} \quad \text { on } \Gamma, \\
& p_{t}=p_{m} \quad \text { on } \Gamma_{\delta}, \\
& \mathbf{u}_{c} \cdot \boldsymbol{\tau}=-R_{\boldsymbol{\tau}}\left[(\mathbf{n} \cdot \nabla) \mathbf{u}_{c}\right] \cdot \boldsymbol{\tau}, \quad \text { on } \Gamma \text {. }
\end{aligned}
$$

where

$$
\nu=\frac{d^{2}}{L^{2}}, \quad \kappa=\frac{k_{t} \mu}{d^{2}}, \quad \kappa_{m}=\frac{k_{m} \mu}{d^{2}}, \quad R_{\tau}=\frac{\sqrt{k_{m} \mu}}{\alpha_{\mathrm{BJ}} L},
$$

are dimensionless quantities.

\section{Asymptotic expansion of the multi-scale model}

We analyze the behaviour of the asymptotic system when the thickness of the capillary wall $\delta$ tends to 0 , assuming that $\kappa_{m}$ is proportional to $\delta$ with a proportionality coefficient $R_{\mathbf{n}}$ that will be defined later on:

$$
\kappa_{m}=\delta R_{\mathbf{n}}
$$

Let us denote by $\eta$ the normal variable to the vessel membrane and by $\theta$ the tangential variable to the vessel wall. With these coordinates, the Laplacian is defined by

$$
\nabla^{2}: \frac{1}{\delta^{2}} \partial_{\eta}^{2}+\frac{1}{\delta(1+\delta \zeta \eta)} \partial_{\eta}+\frac{1}{(1+\delta \zeta \eta)^{2}} \partial_{\theta}^{2}+\partial_{z}^{2}
$$

where $\zeta$ is the curvature of the section. Therefore, the fluid transport equations in the capillary wall and the interface conditions are given by

$$
\begin{aligned}
\left(\partial_{\eta}^{2}+\frac{\delta}{(1+\delta \zeta \eta)} \partial_{\eta}+\frac{\delta^{2}}{(1+\delta \zeta \eta)^{2}} \partial_{\theta}^{2}+\delta^{2} \partial_{z}^{2}\right) p_{m} & =0 & & \text { in } \Omega_{m}, \\
\mathbf{u}_{c} \cdot \mathbf{n} & =-R_{\mathbf{n}} \partial_{\eta} p_{m} & & \text { on } \Gamma \\
\partial_{\eta} p_{m} & =\frac{\kappa}{R_{\mathbf{n}}} \nabla p_{t} \cdot \mathbf{n} & & \text { on } \Gamma_{\delta} \\
p_{c}-\nu\left[(\mathbf{n} \cdot \nabla) \mathbf{u}_{c}\right] \cdot \mathbf{n} & =p_{m} & & \text { on } \Gamma, \\
p_{t} & =p_{m} & & \text { on } \Gamma_{\delta}, \\
\mathbf{u}_{c} \cdot \boldsymbol{\tau} & =-R_{\boldsymbol{\tau}}\left[(\mathbf{n} \cdot \nabla) \mathbf{u}_{c}\right] \cdot \boldsymbol{\tau} & & \text { on } \Gamma
\end{aligned}
$$

We perform an asymptotic expansion of the variables $p_{m}, p_{t}, p_{c}$ and $\mathbf{u}_{c}$ :

$$
\begin{aligned}
p_{m} & =p_{m}^{(0)}+\delta p_{m}^{(1)}+\delta^{2} p_{m}^{(2)}+\ldots \\
p_{t} & =p_{t}^{(0)}+\delta p_{t}^{(1)}+\delta^{2} p_{t}^{(2)}+\ldots \\
p_{c} & =p_{c}^{(0)}+\delta p_{c}^{(1)}+\delta^{2} p_{c}^{(2)}+\ldots \\
\mathbf{u}_{c} & =\mathbf{u}_{c}^{(0)}+\delta \mathbf{u}_{c}^{(1)}+\delta^{2} \mathbf{u}_{c}^{(2)}+\ldots
\end{aligned}
$$


Equating coefficients of $\delta^{0}$ in (S17)-(S22), we obtain the following system of equations

$$
\begin{aligned}
\partial_{\eta}^{2} p_{m}^{(0)} & =0 & & \text { in } \Omega_{m} \\
\mathbf{u}_{c}^{(0)} \cdot \mathbf{n} & =-R_{\mathbf{n}} \partial_{\eta} p_{m}^{(0)} & & \text { on } \Gamma \\
\partial_{\eta} p_{m}^{(0)} & =\frac{\kappa}{R_{\mathbf{n}}} \nabla p_{t}^{(0)} \cdot \mathbf{n} & & \text { on } \Gamma_{\delta} \\
p_{c}^{(0)}-\nu\left[(\mathbf{n} \cdot \nabla) \mathbf{u}_{c}^{(0)}\right] \cdot \mathbf{n} & =p_{m}^{(0)} & & \text { on } \Gamma \\
p_{m}^{(0)} & =p_{t}^{(0)} & & \text { on } \Gamma_{\delta} \\
\mathbf{u}_{c}^{(0)} \cdot \boldsymbol{\tau} & =-R_{\boldsymbol{\tau}}\left[(\mathbf{n} \cdot \nabla) \mathbf{u}_{c}^{(0)}\right] \cdot \boldsymbol{\tau} & & \text { on } \Gamma
\end{aligned}
$$

From (S23) and (S25) we infer

$$
\partial_{\eta} p_{m}^{(0)}=\frac{\kappa}{R_{\mathbf{n}}} \nabla p_{t}^{(0)} \cdot \mathbf{n} \quad \text { in } \Omega_{m},
$$

and then equations (S29) and (S26) leads to

$$
p_{m}^{(0)}=\left(\frac{\kappa}{R_{\mathbf{n}}} \nabla p_{t}^{(0)} \cdot \mathbf{n}\right) \eta+p_{c}^{(0)}-\nu\left[(\mathbf{n} \cdot \nabla) \mathbf{u}_{c}^{(0)}\right] \cdot \mathbf{n} \quad \text { in } \Omega_{m}
$$

Therefore from (S27)

$$
p_{t}^{(0)}=p_{m}^{(0)}(\eta=1)=\frac{\kappa}{R_{\mathbf{n}}} \nabla p_{t}^{(0)} \cdot \mathbf{n}+p_{c}^{(0)}-\nu\left[(\mathbf{n} \cdot \nabla) \mathbf{u}_{c}^{(0)}\right] \cdot \mathbf{n} \quad \text { on } \Gamma_{\delta} .
$$

Let us define by $\Gamma \in \mathbb{R}^{N-1}$ the boundary when $\delta$ goes to 0 , i.e. when the two interfaces $\Gamma_{\delta}$ and $\Gamma$ are superimposed. From equations (S31), (S24) and (S25) we derive formally the boundary conditions for small $\delta$ :

$$
\begin{aligned}
\kappa \nabla p_{t}^{(0)} \cdot \mathbf{n} & =R_{\mathbf{n}}\left(p_{t}^{(0)}-p_{c}^{(0)}+\nu\left[(\mathbf{n} \cdot \nabla) \mathbf{u}_{c}^{(0)}\right] \cdot \mathbf{n}\right) & & \text { on } \Gamma, \\
\mathbf{u}_{c}^{(0)} \cdot \mathbf{n} & =-\kappa \nabla p_{t}^{(0)} \cdot \mathbf{n} & & \text { on } \Gamma, \\
\mathbf{u}_{c}^{(0)} \cdot \boldsymbol{\tau} & =-R_{\boldsymbol{\tau}}\left[(\mathbf{n} \cdot \nabla) \mathbf{u}_{c}^{(0)}\right] \cdot \boldsymbol{\tau} & & \text { on } \Gamma .
\end{aligned}
$$

Conditions (S32)-(S33) can be rewritten as

$$
\mathbf{u}_{t} \cdot \mathbf{n}=\mathbf{u}_{c} \cdot \mathbf{n}=R_{\mathbf{n}}\left(p_{c}-p_{t}-\nu\left[(\mathbf{n} \cdot \nabla) \mathbf{u}_{c}\right] \cdot \mathbf{n}\right) \quad \text { on } \Gamma .
$$

Equation (S35) is similar to Starling's law, that is the most widely used equation in literature to model flux transport across the vessel wall $[5,6]$ and reads

$$
\mathbf{u}_{c} \cdot \mathbf{n}=L_{p}\left(p_{c}-p_{t}-\sigma\left(\pi_{c}-\pi_{t}\right)\right),
$$

where $L_{p}$ is the vascular permeability, $\sigma$ is the osmotic reflection coefficient $(\sigma \in(0,1))$ that expresses the glycocalyx filter function through the endothelial wall and $\left(\pi_{c}-\pi_{t}\right)$ is the oncotic pressure difference between the capillaries and the interstitium. However, the latter can be considered negligible compared to the interstitial fluid pressure difference in tumors $[7,8]$. Moreover, the viscous term in equation (S35) is usually neglected but it is necessary to guarantee the wellposedness of the problem and does not change the physical meaning since it is based on the balance of the normal forces [9].

The relation between $R_{\mathbf{n}}$ and $L_{p}$ is given thanks to the nondimensionalization of equation (S36):

$$
R_{\mathbf{n}}=\frac{L_{p} L \mu}{d^{2}}
$$

Therefore, our model is composed by equations (2), (2) in the respective regions of the domain $\Omega_{t}$ and $\Omega_{c}$ and by the interface conditions (S32)-(S34) on $\Gamma$. 


\section{S2: Derivation of the macro-scale models}

We derive the macroscopic models using formal two-scale homogenisation according to the magnitude of the permeability of the vessel wall and of the interstitial hydraulic conductivity, namely

- Model 1: $\gamma=0, \eta=0$;

- Model 2: $\gamma=1, \eta=0$;

- Model 3: $\gamma=2, \eta=1$.

The differential operators are then

$$
\nabla=\nabla_{\mathbf{x}}+\frac{1}{\varepsilon} \nabla_{\mathbf{y}}, \quad \nabla^{2}=\nabla_{\mathbf{x}}^{2}+\frac{2}{\varepsilon} \nabla_{\mathbf{x}} \cdot \nabla_{\mathbf{y}}+\frac{1}{\varepsilon^{2}} \nabla_{\mathbf{y}}^{2}
$$

The fluid transport in the tumor tissue can be then written as follows:

$$
\begin{aligned}
\varepsilon^{3} \bar{\nu} \nabla_{\mathbf{x}}^{2} \mathbf{u}_{c}+2 \varepsilon^{2} \bar{\nu} \nabla_{\mathbf{y}} \cdot \nabla_{\mathbf{x}} \mathbf{u}_{c}+\varepsilon \bar{\nu} \nabla_{\mathbf{y}}^{2} \mathbf{u}_{c} & =\varepsilon \nabla_{\mathbf{x}} p_{c}+\nabla_{\mathbf{y}} p_{c} & & \text { in } \Omega_{c}^{\varepsilon} \times Y_{c}, \\
\varepsilon \nabla_{\mathbf{x}} \cdot \mathbf{u}_{c}+\nabla_{\mathbf{y}} \cdot \mathbf{u}_{c} & =0 & & \text { in } \Omega_{c}^{\varepsilon} \times Y_{c}, \\
\varepsilon^{2} \nabla_{\mathbf{x}}^{2} p_{t}+2 \varepsilon \nabla_{\mathbf{x}} \cdot \nabla_{\mathbf{y}} p_{t}+\nabla_{\mathbf{y}}^{2} p_{t} & =0 & & \text { in } \Omega_{t}^{\varepsilon} \times Y_{t} .
\end{aligned}
$$

The interface conditions vary according to the value of $\gamma$ and $\eta$ :

$$
\begin{aligned}
-\varepsilon^{2} \bar{\nu}\left[\left(\mathbf{n} \cdot \nabla_{\mathbf{x}}\right) \mathbf{u}_{c}\right] \cdot \mathbf{n}-\varepsilon \bar{\nu}\left[\left(\mathbf{n} \cdot \nabla_{\mathbf{y}}\right) \mathbf{u}_{c}\right] \cdot \mathbf{n}+p_{c}-p_{t} & =\frac{1}{\varepsilon^{\gamma} \bar{R}_{\mathbf{n}}} \mathbf{u}_{c} \cdot \mathbf{n} & & \text { on } \Gamma^{\varepsilon} \times \Gamma_{Y}, \\
-\varepsilon \nu\left[\left(\mathbf{n} \cdot \nabla_{\mathbf{x}}\right) \mathbf{u}_{c}\right] \cdot \boldsymbol{\tau}-\nu\left[\left(\mathbf{n} \cdot \nabla_{\mathbf{y}}\right) \mathbf{u}_{c}\right] \cdot \boldsymbol{\tau} & =\varepsilon \frac{\nu}{R_{\boldsymbol{\tau}}} \mathbf{u}_{c} \cdot \boldsymbol{\tau} & & \text { on } \Gamma^{\varepsilon} \times \Gamma_{Y}, \\
-\varepsilon^{\eta} \bar{\kappa} \nabla_{\mathbf{x}} p_{t} \cdot \mathbf{n}-\varepsilon^{\eta-1} \bar{\kappa} \nabla_{\mathbf{y}} p_{t} \cdot \mathbf{n} & =\mathbf{u}_{c} \cdot \mathbf{n} & & \text { on } \Gamma^{\varepsilon} \times \Gamma_{Y} .
\end{aligned}
$$

Case 1: permeable vessel walls i.e. $\gamma=0, \eta=0$.

When $\gamma=0$, equation (S41) reads as

$$
-\varepsilon^{2} \bar{\nu}\left[\left(\mathbf{n} \cdot \nabla_{\mathbf{x}}\right) \mathbf{u}_{c}\right] \cdot \mathbf{n}-\varepsilon \bar{\nu}\left[\left(\mathbf{n} \cdot \nabla_{\mathbf{y}}\right) \mathbf{u}_{c}\right] \cdot \mathbf{n}+p_{c}-p_{t}=\frac{1}{\bar{R}_{\mathbf{n}}} \mathbf{u}_{c} \cdot \mathbf{n} \quad \text { on } \Gamma^{\varepsilon} \times \Gamma_{Y}
$$

\section{Identifying the terms of order $\varepsilon^{0}$.}

In the interstitium, equating coefficients of $\varepsilon^{0}$ in (S40) and (S43) gives

$$
\begin{aligned}
\nabla_{\mathbf{y}}^{2} p_{t}^{(0)} & =0 & & \text { in } \Omega_{t}^{\varepsilon} \times Y_{t}, \\
\bar{\kappa} \nabla_{\mathbf{y}} p_{t}^{(0)} \cdot \mathbf{n} & =0 & & \text { on } \Gamma^{\varepsilon} \times \Gamma_{Y},
\end{aligned}
$$

with $p_{t}^{(0)}$ periodic in $\mathbf{y}$. Therefore $p_{t}^{(0)}$ does not depend on the local scale, i.e. $p_{t}^{(0)}=p_{t}^{(0)}(\mathbf{x})$. Equating coeffiecients of $\varepsilon^{0}$ in the capillaries gives:

$$
\begin{aligned}
\nabla_{\mathbf{y}} p_{c}^{(0)} & =0 & & \text { in } \Omega_{c}^{\varepsilon} \times Y_{c}, \\
\nabla_{\mathbf{y}} \cdot \mathbf{u}_{c}^{(0)} & =0 & & \text { in } \Omega_{c}^{\varepsilon} \times Y_{c}, \\
\mathbf{u}_{c}^{(0)} \cdot \mathbf{n} & =\bar{R}_{\mathbf{n}}\left(p_{c}^{(0)}-p_{t}^{(0)}\right) & & \text { on } \Gamma^{\varepsilon} \times \Gamma_{Y}, \\
{\left[\left(\mathbf{n} \cdot \nabla_{\mathbf{y}}\right) \mathbf{u}_{c}^{(0)}\right] \cdot \boldsymbol{\tau} } & =0 & & \text { on } \Gamma^{\varepsilon} \times \Gamma_{Y},
\end{aligned}
$$

plus periodic boundary conditions on $p_{c}^{(0)}$ and $\mathbf{u}_{c}^{(0)}$ in $\mathbf{y}$. Integrating equation (S48) we get the following condition on the pressure $p_{c}^{(0)}$ :

$$
\int_{Y_{c}} \nabla_{\mathbf{y}} \cdot \mathbf{u}_{c}^{(0)} \mathrm{d} V=\int_{\Gamma_{Y}} \bar{R}_{\mathbf{n}}\left(p_{c}^{(0)}-p_{t}^{(0)}\right) \mathrm{d} S=0 \quad \Rightarrow \quad p_{c}^{(0)}=p_{t}^{(0)} \text { on } \Gamma_{Y},
$$


since $p_{t}^{(0)}$ is a constant with respect to $\mathbf{y}$. Therefore, $p_{c}^{(0)}$ depends on the macroscale only and it is equal to $p_{t}^{(0)}(\mathbf{x})$. Moreover, condition (S49) becomes

$$
\mathbf{u}_{c}^{(0)} \cdot \mathbf{n}=0 \quad \text { on } \Gamma^{\varepsilon} \times \Gamma_{Y} .
$$

\section{Identifying the terms of order $\varepsilon^{1}$.}

Equating coefficients of $\varepsilon^{1}$ in (S40) and (S43) yields

$$
\begin{aligned}
\nabla_{\mathbf{y}}^{2} p_{t}^{(1)} & =0 & & \text { in } \Omega_{t}^{\varepsilon} \times Y_{t}, \\
-\bar{\kappa} \nabla_{\mathbf{x}} p_{t}^{(0)} \cdot \mathbf{n}-\bar{\kappa} \nabla_{\mathbf{y}} p_{t}^{(1)} \cdot \mathbf{n} & =\mathbf{u}_{c}^{(0)} \cdot \mathbf{n} & & \text { on } \Gamma^{\varepsilon} \times \Gamma_{Y},
\end{aligned}
$$

where $\mathbf{u}_{c}^{(0)} \cdot \mathbf{n}=0$. We exploit the linearity of system (S52)-(S53) and propose a solution of the form

$$
p_{t}^{(1)}=-\sum_{j=1}^{N} \mathrm{G}^{j}\left(\nabla_{\mathbf{x}} p_{t}^{(0)} \cdot \mathbf{e}_{j}\right)+\bar{p}_{t}^{(1)},
$$

where $\mathbf{G}^{j}=\mathbf{G}^{j}(\mathbf{y})$ satisfies the cell problem (7) for $j=1, \ldots, N$.

Equating coeffiecients of $\varepsilon^{1}$ in the capillaries yields:

$$
\begin{aligned}
\bar{\nu} \nabla_{\mathbf{y}}^{2} \mathbf{u}_{c}^{(0)} & =\nabla_{\mathbf{x}} p_{c}^{(0)}+\nabla_{\mathbf{y}} p_{c}^{(1)} & & \text { in } \Omega_{c}^{\varepsilon} \times Y_{c}, \\
\nabla_{\mathbf{x}} \cdot \mathbf{u}_{c}^{(0)}+\nabla_{\mathbf{y}} \cdot \mathbf{u}_{c}^{(1)} & =0 & & \text { in } \Omega_{c}^{\varepsilon} \times Y_{c}, \\
\mathbf{u}_{c}^{(1)} \cdot \mathbf{n} & =\bar{R}_{\mathbf{n}}\left(p_{c}^{(1)}-p_{t}^{(1)}-\bar{\nu}\left[\left(\mathbf{n} \cdot \nabla_{\mathbf{y}}\right) \cdot \mathbf{u}_{c}^{(0)}\right] \cdot \mathbf{n}\right) & & \text { on } \Gamma^{\varepsilon} \times \Gamma_{Y}, \\
-R_{\boldsymbol{\tau}}\left[\left(\mathbf{n} \cdot \nabla_{\mathbf{y}}\right) \mathbf{u}_{c}^{(1)}\right] \cdot \mathbf{n} \cdot \boldsymbol{\tau} & =\mathbf{u}_{c}^{(0)} \cdot \boldsymbol{\tau}+R_{\boldsymbol{\tau}}\left[\left(\mathbf{n} \cdot \nabla_{\mathbf{x}}\right) \mathbf{u}_{c}^{(0)}\right] \cdot \boldsymbol{\tau} & & \text { on } \Gamma^{\varepsilon} \times \Gamma_{Y} .
\end{aligned}
$$

We exploit the linearity of the system composed by (S55)-(S48)-(S51)-(S50) and propose a solution of the form:

$$
\begin{aligned}
& \mathbf{u}_{c}^{(0)}=-\sum_{j=1 j}^{N} \mathbf{W}^{j}\left(\nabla_{\mathbf{x}} p_{c}^{(0)} \cdot \mathbf{e}_{j}\right) \\
& p_{c}^{(1)}=-\sum_{j=1}^{N} P^{j}\left(\nabla_{\mathbf{x}} p_{c}^{(0)} \cdot \mathbf{e}_{j}\right)+\bar{p}^{(1)}(\mathbf{x}),
\end{aligned}
$$

where $\left(\mathbf{W}^{j}, P^{j}\right)$ solve the cell problem (8) for $j=1, \ldots N$. Integrating (S59) over $Y_{c}$, we find the leading order for the velocity $\mathbf{u}_{c}^{(0)}$ :

$$
\left\langle\mathbf{u}_{c}^{(0)}\right\rangle_{Y_{c}}=-\mathbf{E} \nabla_{\mathbf{x}} p_{c}^{(0)}
$$

where $\mathbf{E}$ is defined in (9).

\section{Identifying the terms of order $\varepsilon^{2}$.}

Equating coefficients of $\varepsilon^{2}$ in (S40) and (S43) gives:

$$
\begin{array}{rlrl}
\nabla_{\mathbf{x}}^{2} p_{t}^{(0)}+2 \nabla_{\mathbf{x}} \cdot \nabla_{\mathbf{y}} p_{t}^{(1)}+\nabla_{\mathbf{y}}^{2} p_{t}^{(2)} & =0 & & \text { in } \Omega_{t}^{\varepsilon} \times Y_{t}, \\
-\bar{\kappa} \nabla_{\mathbf{x}} p_{t}^{(1)} \cdot \mathbf{n}-\bar{\kappa} \nabla_{\mathbf{y}} p_{t}^{(2)} \cdot \mathbf{n}=\mathbf{u}_{c}^{(1)} \cdot \mathbf{n} & & \text { on } \Gamma^{\varepsilon} \times \Gamma_{Y} .
\end{array}
$$

Integrating (S62) we obtain the equation for the leading order of the interstitial pressure:

$$
\nabla_{\mathbf{x}} \cdot\left(\left(\bar{\kappa} \mathbf{K}+\frac{\left|Y_{c}\right|}{\left|Y_{t}\right|} \mathbf{E}\right) \nabla_{\mathbf{x}} p_{t}^{(0)}\right)=0
$$

where $\mathbf{K}$ and $\mathbf{E}$ are defined in (9). 
Case 2: weakly permeable vessel walls i.e. $\gamma=1, \eta=0$.

When $\gamma=1$, equation (S41) becomes

$$
-\varepsilon^{2} \bar{\nu}\left[\left(\mathbf{n} \cdot \nabla_{\mathbf{x}}\right) \mathbf{u}_{c}\right] \cdot \mathbf{n}-\varepsilon \bar{\nu}\left[\left(\mathbf{n} \cdot \nabla_{\mathbf{y}}\right) \mathbf{u}_{c}\right] \cdot \mathbf{n}+p_{c}-p_{t}=\frac{1}{\varepsilon \bar{R}_{\mathbf{n}}} \mathbf{u}_{c} \cdot \mathbf{n} \quad \text { on } \Gamma^{\varepsilon} \times \Gamma_{Y} .
$$

This case has been previously addressed [10,9]. We write here the formal derivation of the macroscale model for the sake of completeness. Equations in the interstitium are the same as the ones in the previous case, while equations (S49) and (S57) take a different form in the capillaries. Nevertheless, the cell problems defined in (7) and (8) hold.

\section{Identifying the terms of order $\varepsilon^{0}$.}

As for the previous case, $p_{t}^{(0)}$ in the interstitium does not depend on the micro-scale, i.e. $p_{t}^{(0)}=p_{t}^{(0)}(\mathbf{x})$. The interface conditions for the variables $p_{c}^{(0)}$ and $\mathbf{u}_{c}^{(0)}$ read as follows:

$$
\begin{aligned}
\mathbf{u}_{c}^{(0)} \cdot \mathbf{n} & =0, & & \text { on } \Gamma^{\varepsilon} \times \Gamma_{Y}, \\
{\left[\left(\mathbf{n} \cdot \nabla_{\mathbf{y}}\right) \mathbf{u}_{c}^{(0)}\right] \cdot \boldsymbol{\tau} } & =0 & & \text { on } \Gamma^{\varepsilon} \times \Gamma_{Y} .
\end{aligned}
$$

Equating coefficients of $\varepsilon^{0}$ in the capillaries, equations (S47)-(S48)-(S66)-(S67) hold. Therefore, the leading order of the pressure in the capillaries depends only on the macro-scale $p_{c}^{(0)}=p_{c}^{(0)}(\mathbf{x})$.

\section{Identifying the terms of order $\varepsilon^{1}$.}

In the interstitium, the same results found in the previous case hold.

Equating coefficients of $\varepsilon^{1}$ in the capillaries, yields

$$
\begin{aligned}
\bar{\nu} \nabla_{\mathbf{y}}^{2} \mathbf{u}_{c}^{(0)} & =\nabla_{\mathbf{x}} p_{c}^{(0)}+\nabla_{\mathbf{y}} p_{c}^{(1)} & & \text { in } \Omega_{c}^{\varepsilon} \times Y_{c}, \\
\nabla_{\mathbf{x}} \cdot \mathbf{u}_{c}^{(0)}+\nabla_{\mathbf{y}} \cdot \mathbf{u}_{c}^{(1)} & =0 & & \text { in } \Omega_{c}^{\varepsilon} \times Y_{c}, \\
\mathbf{u}_{c}^{(1)} \cdot \mathbf{n} & =\bar{R}_{\mathbf{n}}\left(p_{c}^{(0)}-p_{t}^{(0)}\right) & & \text { on } \Gamma^{\varepsilon} \times \Gamma_{Y}, \\
-R_{\boldsymbol{\tau}}\left[\left(\mathbf{n} \cdot \nabla_{\mathbf{y}}\right) \mathbf{u}_{c}^{(1)}\right] \cdot \mathbf{n} \cdot \boldsymbol{\tau} & =\mathbf{u}_{c}^{(0)} \cdot \boldsymbol{\tau}+R_{\boldsymbol{\tau}}\left[\left(\mathbf{n} \cdot \nabla_{\mathbf{x}}\right) \mathbf{u}_{c}^{(0)}\right] \cdot \boldsymbol{\tau} & & \text { on } \Gamma^{\varepsilon} \times \Gamma_{Y} .
\end{aligned}
$$

Exploiting the linearity of this system, we propose a solution of the same type of (S59) and (S60) where $\left(\mathbf{W}^{j}, \mathrm{P}^{j}\right)$ solve the cell problem defined in (8).

\section{Identifying the terms of order $\varepsilon^{2}$.}

In the instersitial domain, equating coefficients of $\varepsilon^{2}$ yields equations (S62) and (S63). We obtain the equations for the leading order by integrating (S59), (S70) in the capillaries, (S62) and (S63) in the interstitium:

$$
\begin{aligned}
\nabla_{\mathbf{x}} \cdot\left(\bar{\kappa} \mathbf{K} \nabla_{\mathbf{x}} p_{t}^{(0)}\right) & =\frac{\bar{R}_{\mathbf{n}}\left|\Gamma_{Y}\right|}{\left|Y_{t}\right|}\left(p_{t}^{(0)}-p_{c}^{(0)}\right), \\
\nabla_{\mathbf{x}} \cdot\left(\mathbf{E} \nabla_{\mathbf{x}} p_{c}^{(0)}\right) & =\frac{\bar{R}_{\mathbf{n}}\left|\Gamma_{Y}\right|}{\left|Y_{c}\right|}\left(p_{c}^{(0)}-p_{t}^{(0)}\right) .
\end{aligned}
$$

Case 3: weakly permeable walls and weakly interstitial hydraulic connectivity i.e. $\gamma=2, \eta=1$.

When $\gamma=2$ and $\eta=1$, equations (S41)-(S43) become

$$
\begin{array}{rlrl}
-\varepsilon^{2} \bar{\nu}\left[\left(\mathbf{n} \cdot \nabla_{\mathbf{x}}\right) \mathbf{u}_{c}\right] \cdot \mathbf{n}-\varepsilon \bar{\nu}\left[\left(\mathbf{n} \cdot \nabla_{\mathbf{y}}\right) \mathbf{u}_{c}\right] \cdot \mathbf{n}+p_{c}-p_{t} & =\frac{1}{\varepsilon^{2} \bar{R}_{\mathbf{n}}} \mathbf{u}_{c} \cdot \mathbf{n} \\
-\varepsilon \bar{\kappa} \nabla_{\mathbf{x}} p_{t} \cdot \mathbf{n}-\bar{\kappa} \nabla_{\mathbf{y}} p_{t} \cdot \mathbf{n} & =\mathbf{u}_{c} \cdot \mathbf{n} & & \text { on } \Gamma^{\varepsilon} \times \Gamma_{Y}, \\
& & \text { on } \Gamma^{\varepsilon} \times \Gamma_{Y} .
\end{array}
$$




\section{Identifying the terms of order $\varepsilon^{0}$.}

Equating the coefficients of $\varepsilon^{(0)}$, equation (S45) holds in the interstitium and (S47)-(S48) hold in the capillaries.

The interface conditions for the variables $p_{t}^{(0)}, p_{c}^{(0)}$ and $\mathbf{u}_{c}^{(0)}$ read as follows:

$$
\begin{aligned}
-\bar{\kappa} \nabla_{\mathbf{y}} p_{t}^{(0)} \cdot \mathbf{n} & =\mathbf{u}_{c}^{(0)} \cdot \mathbf{n}, & & \text { on } \Gamma^{\varepsilon} \times \Gamma_{Y}, \\
\mathbf{u}_{c}^{(0)} \cdot \mathbf{n} & =0, & & \text { on } \Gamma^{\varepsilon} \times \Gamma_{Y}, \\
{\left[\left(\mathbf{n} \cdot \nabla_{\mathbf{y}}\right) \mathbf{u}_{c}^{(0)}\right] \cdot \boldsymbol{\tau} } & =0 & & \text { on } \Gamma^{\varepsilon} \times \Gamma_{Y} .
\end{aligned}
$$

Since $\mathbf{u}_{c}^{(0)} \cdot \mathbf{n}=0$ on $\Gamma^{\varepsilon} \times \Gamma_{Y}, p_{t}^{(0)}$ in the interstitium does not depend on the micro-scale, i.e. $p_{t}^{(0)}=p_{t}^{(0)}(\mathbf{x})$. Equating coefficients of $\varepsilon^{0}$ in the capillaries, equations (S47)-(S48)-(S76)-(S77) hold. Therefore, the leading order of the pressure in the capillaries depends only on the macro-scale $p_{c}^{(0)}=p_{c}^{(0)}(\mathbf{x})$.

\section{Identifying the terms of order $\varepsilon^{1}$.}

Equating coefficients of $\varepsilon^{1}$, equation (S52) holds in the interstitium while equations (S55)-(S56) hold in the capillaries. The interface conditions for the variables $p_{t}^{(1)}, p_{c}^{(1)}$ and $\mathbf{u}_{c}^{(1)}$ read as follows:

$$
\begin{aligned}
-\bar{\kappa} \nabla_{\mathbf{x}} p_{t}^{(0)} \cdot \mathbf{n}-\bar{\kappa} \nabla_{\mathbf{y}} p_{t}^{(1)} \cdot \mathbf{n} & =\mathbf{u}_{c}^{(1)} \cdot \mathbf{n}, & & \text { on } \Gamma^{\varepsilon} \times \Gamma_{Y}, \\
\mathbf{u}_{c}^{(1)} \cdot \mathbf{n} & =0, & & \text { on } \Gamma^{\varepsilon} \times \Gamma_{Y}, \\
-R_{\boldsymbol{\tau}}\left[\left(\mathbf{n} \cdot \nabla_{\mathbf{y}}\right) \mathbf{u}_{c}^{(1)}\right] \cdot \mathbf{n} \cdot \boldsymbol{\tau} & =\mathbf{u}_{c}^{(0)} \cdot \boldsymbol{\tau}+R_{\boldsymbol{\tau}}\left[\left(\mathbf{n} \cdot \nabla_{\mathbf{x}}\right) \mathbf{u}_{c}^{(0)}\right] \cdot \boldsymbol{\tau} & & \text { on } \Gamma^{\varepsilon} \times \Gamma_{Y} .
\end{aligned}
$$

In the interstitium, since $\mathbf{u}_{c}^{(1)} \cdot \mathbf{n}=0$ on $\Gamma^{\varepsilon} \times \Gamma_{Y}$, the same results for $p_{t}^{(1)}$ found in the previous cases hold.

In the capillaries, we exploit the linearity of the system (S55)-(S48)-(S76)-(S77) and propose a solution for $\mathbf{u}_{c}^{(0)}$ and $p_{c}^{(1)}$ of the same type of (S59) and (S60) where $\left(\mathbf{W}^{j}, \mathrm{P}^{j}\right)$ solve the cell problem defined in (8).

\section{Identifying the terms of order $\varepsilon^{2}$.}

Equating coefficients of $\varepsilon^{2}$ in the interstitium, we obtain (S62) and the following interface condition:

$$
-\bar{\kappa} \nabla_{\mathbf{x}} p_{t}^{(1)} \cdot \mathbf{n}-\bar{\kappa} \nabla_{\mathbf{y}} p_{t}^{(2)} \cdot \mathbf{n}=\mathbf{u}_{c}^{(2)} \cdot \mathbf{n}, \quad \text { on } \Gamma^{\varepsilon} \times \Gamma_{Y} .
$$

Equating coefficients of $\varepsilon^{2}$ in (S42), the following interface condition holds:

$$
\mathbf{u}_{c}^{(2)} \cdot \mathbf{n}=\bar{R}_{\mathbf{n}}\left(p_{c}^{(0)}-p_{t}^{(0)}\right), \quad \text { on } \Gamma^{\varepsilon} \times \Gamma_{Y} .
$$

Integrating equations (S62) and (S81) we obtain the equation for the leading order of the interstitial pressure:

$$
\nabla_{\mathbf{x}} \cdot\left(\bar{\kappa} \mathbf{K} \nabla_{\mathbf{x}} p_{t}^{(0)}\right)=\frac{\bar{R}_{\mathbf{n}}\left|\Gamma_{Y}\right|}{\left|Y_{t}\right|}\left(p_{t}^{(0)}-p_{c}^{(0)}\right) .
$$

Integrating equation (S59) and (S79), we obtain the leading order for the pressure in the capillaries:

$$
\nabla \cdot\left(\mathbf{E} \nabla p_{c}\right)=0
$$



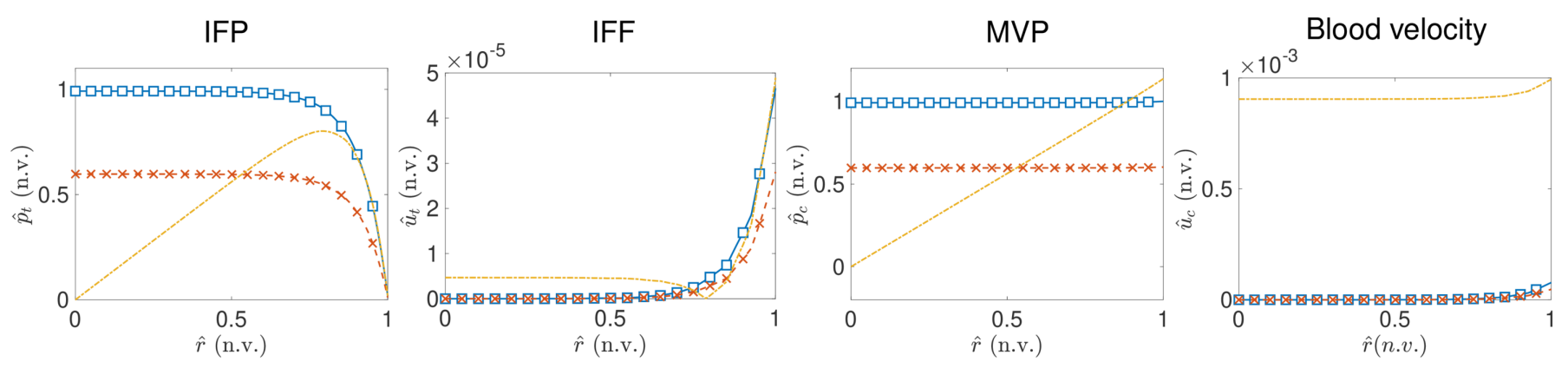

Dirichlet - $\star$ Neumann 1 ----Neumann 2

Figure S1: Normalized values (n.v.) of the interstitial fluid pressure (IFP) and flow (IFF), of the microvascular pressure (MVP) and of the blood velocity as functions of the normalized radius $\hat{r}$ varying the boundary conditions. The microstructure considered in this case corresponds to Fig 3c.

\section{References}

[1] M. Discacciati and A. Quarteroni, "Navier-Stokes/darcy coupling: Modeling, analysis, and numerical approximation," Rev. Mat. Complut., vol. 22, July 2009.

[2] W. J. Layton, F. Schieweck, and I. Yotov, “Coupling Fluid Flow with Porous Media Flow," SIAM J. Numer. Anal., vol. 40, pp. 2195-2218, Jan. 2002.

[3] G. S. Beavers and D. D. Joseph, "Boundary conditions at a naturally permeable wall," J. Fluid. Mech, vol. 30, pp. 197207, Oct. 1967.

[4] P. G. Saffman, "On the Boundary Condition at the Surface of a Porous Medium," Stud. Appl. Math., vol. 50, pp. 93-101, June 1971.

[5] R. K. Jain and L. T. Baxter, "Mechanisms of heterogeneous distribution of monoclonal antibodies and other macromolecules in tumors: Significance of elevated interstitial pressure," Cancer Res., vol. 48, pp. 7022-7032, Dec. 1988.

[6] J. W. Baish, P. A. Netti, and R. K. Jain, "Transmural coupling of fluid flow in microcirculatory network and interstitium in tumors," Microvasc. Res., vol. 53, pp. 128-141, Mar. 1997.

[7] R. K. Jain, "Transport of molecules in the tumor interstitium: A review," Cancer Res., vol. 47, pp. 3039-3051, June 1987.

[8] C. Voutouri and T. Stylianopoulos, "Evolution of osmotic pressure in solid tumors," J Biomech., vol. 47, pp. 3441-3447, Nov. 2014.

[9] R. Penta, D. Ambrosi, and A. Quarteroni, "Multiscale homogenization for fluid and drug transport in vascularized malignant tissues," Math. Models Methods Appl. Sci., vol. 25, pp. 79-108, Jan. 2015.

[10] R. J. Shipley and S. J. Chapman, "Multiscale Modelling of Fluid and Drug Transport in Vascular Tumours," Bull Math Biol, vol. 72, pp. 1464-1491, Aug. 2010. 